\title{
QUANTUM SYMMETRIC PAIRS AND REPRESENTATIONS OF DOUBLE AFFINE HECKE ALGEBRAS OF TYPE $C^{\vee} C_{n}$
}

\author{
DAVID JORDAN AND XIAOGUANG MA
}

\begin{abstract}
We build representations of the affine and double affine braid groups and Hecke algebras of type $C^{\vee} C_{n}$, based upon the theory of quantum symmetric pairs $(\mathbf{U}, \mathbf{B})$. In the case $\mathbf{U}=\mathcal{U}_{\mathfrak{q}}\left(\mathfrak{g l}_{N}\right)$, our constructions provide a quantization of the representations constructed by Etingof, Freund and $\mathrm{Ma}$ in [EFM], and also a type $C^{\vee} C_{n}$ generalization of the results in [J].
\end{abstract}

\section{INTRODUCTION}

In [Ch], Ivan Cherednik introduced the double affine Hecke algebra (abbreviated DAHA, also known as the Cherednik algebra), as a generalization of the affine Hecke algebra (AHA) associated to an affine root system. The DAHA is a quotient of the group algebra of the double affine braid group by additional Hecke relations. Cherednik used these algebras to prove Macdonald's constant term conjecture for Macdonald polynomials. In [ $\underline{\underline{S}}$, Sahi constructed a six-parameter DAHA associated to the root system $C^{\vee} C_{n}$, and used it to analyze the non-symmetric Macdonald and Koornwinder polynomials.

The degenerate affine Hecke algebra (dAHA) of a Coxeter group was defined by Drinfeld and Lusztig (Dri], Lus $)$. It is a certain multi-parameter deformation of the smash product of the group algebra of the Coxeter group with the coordinate ring of its reflection representation. The degenerate double affine Hecke algebra (dDAHA) of a root system was introduced by Cherednik (see $\mathrm{Ch}$ ). It is a certain multi-parameter deformation of the smash product of the affine Weyl group with the coordinate ring of its reflection representation. The relationship between these algebras and their non-degenerate counterparts is analogous to that between $\mathcal{U}(\mathfrak{g})$ and $\mathcal{U}_{\mathrm{q}}(\mathfrak{g})$ : the former may be recovered from the latter by taking quasi-classical limits with respect to the defining parameters.

Motivated by conformal field theory, Arakawa and Suzuki ([AS]) constructed a functor from the category of Harish-Chandra $\mathcal{U}\left(\mathfrak{g l}_{N}\right)$-bimodules to the category of representations of the dAHA of type $A_{n}$ for each $n \geq 1$. This construction was extended to the dDAHA of type $A_{n}$ by Calaque, Enriquez, and Etingof in CEE, using the theory of ad-equivariant $D$-modules on the algebraic group $G=\mathrm{GL}_{N}$.

In [EFM], these constructions were extended to encompass $B C_{n}$ root systems. More precisely, they considered the symmetric pair of Lie algebras $(\mathfrak{g}, \mathfrak{k})=\left(\mathfrak{g l}_{N}, \mathfrak{g l}_{p} \times\right.$ $\mathfrak{g l}_{q} \sqrt{1}$ associated to the real symmetric pair $(G, K)=(U(N), U(p) \times U(q))$. For each $n$, there were constructed functors from the category of Harish-Chandra modules for

1991 Mathematics Subject Classification. Primary 17B37; Secondary 20 C08.

Key words and phrases. Quantum D-modules, double affine Hecke algebras.

1 all Lie algebras are over $\mathbb{C}$, and $N=p+q$. 
$(G, K)$ to the representations of the dAHA, and from the category of $K$-equivariant $D$-modules on $G / K$ to the representations of the dDAHA of type $B C_{n}$.

In [J], the constructions of [CEE were quantized to encompass the theory of quantum groups, and the non-degenerate DAHA's of type $A_{n}$. Namely, for a quasi-triangular Hopf algebra $\mathbf{U}$, an integer $n \geq 1$, and $V \in \mathbf{U}$-mod, there were constructed functors from the category of $\mathbf{U}$-modules to the category of representations of the affine braid group, and from the category ad-equivariant quantum $D_{\mathbf{U}}$-modules to the representations of the double affine braid group. In case the braiding on $V$ satisfies a Hecke relation, the functors take values in representations of the AHA and DAHA, respectively. Moreover it was shown that in the case $\mathbf{U}=\mathcal{U}_{\mathrm{q}}\left(\mathfrak{s l}_{N}\right)$, the quasiclassical limit $\mathrm{q} \mapsto 1$ recovers the construction of [CEE].

In this paper, we quantize the constructions of [EFM], by appealing to the theory of quantum symmetric pairs, as pioneered by Letzter [L1, L2], and developed further in [DS, Kol, $\mathrm{OS}$, among others. To a simple Lie algebra $\mathfrak{g}$ and an involution $\theta: \mathfrak{g} \rightarrow \mathfrak{g}$ is associated the (classical) symmetric pair $\left(\mathfrak{g}, \mathfrak{g}^{\theta}\right)$. Here $\mathfrak{g}^{\theta}$ is the subalgebra of $\mathfrak{g}$ whose elements are fixed by $\theta$. The quantum analogue of $\mathcal{U}\left(\mathfrak{g}^{\theta}\right)$ is a left (alternatively, right) coideal subalgebra $\mathbf{B} \subset \mathcal{U}_{\mathrm{q}}(\mathfrak{g})$, which specializes to $\mathcal{U}\left(\mathfrak{g}^{\theta}\right)$ as $\mathrm{q} \rightarrow 1$. The pair $\left(\mathcal{U}_{\mathrm{q}}(\mathfrak{g}), \mathbf{B}\right)$ is called a quantum symmetric pair.

For the simple Lie algebras, such pairs were explicitly described by Letzter ([L1, L2]): interestingly, it was shown that in the case of $\left(\mathfrak{g l}_{N}, \mathfrak{g l}_{p} \times \mathfrak{g l}_{q}\right)$, there is a not a unique quantization, but rather a one-parameter family, $\left\{\mathbf{B}_{\sigma}\right\}_{\sigma \in \mathbb{C}}$, of subalgebras, essentially because the involution $\theta$ is replaced by a one-parameter family of automorphisms of $\mathcal{U}_{\mathbf{q}}(\mathfrak{g})$ (see [L1, p. 50). In this case, the algebras $\mathbf{B}_{\sigma}$ are known as quantum Grassmannians, and were first introduced by Dijkhuizen, Noumi and Sugitani in the paper [DNS.

Basic algebraic properties of quantum symmetric pairs, and their connection to the so-called reflection equations were established in [KoSt]. In particular, it was explained there how so-called Noumi coideal subalgebras can be constructed canonically, starting from a character of the braided dual, $\mathbf{A}$, of $\mathbf{U}$. In the case $\mathbf{U}=\mathcal{U}_{\mathrm{q}}\left(\mathfrak{g l}_{N}\right)$, characters of the reflection equation algebra were classified by Mudrov Mud, and it was explained in KoSt how to extend these to its localization, A.

Our general setup is as follows. We let $\mathbf{U}$ be a quasitriangular Hopf algebra. We choose a character $f: \mathbf{A} \rightarrow \mathbb{C}$, and denote by $\mathbf{B}_{f} \subset \mathbf{U}$ the corresponding left Noumi coideal subalgebra. We further choose a character $\chi: \mathbf{B}_{f} \rightarrow \mathbb{C}$. For each $n \geq 1$, we construct with this data a functor from the category of $\mathbf{U}$-modules to representations of the affine braid group of type $C^{\vee} C_{n}$. Next, we choose a second character $g: \mathbf{A} \rightarrow \mathbb{C}$, and denote by $\mathbf{B}_{g}^{\prime}$ the corresponding right Noumi coideal subalgebra. We let $\chi^{\prime}: \mathbf{B}_{g}^{\prime} \rightarrow \mathbb{C}$ be a character. To this data, we associate a functor from the category of $D_{\mathbf{U}}$-modules (satisfying some technical conditions) to the category to representations of the double affine braid group of type $C^{\vee} C_{n}$, by analogy with [EFM]. Our main results are Theorems 5.1, 6.10, 8.1, and 9.1, where we detail the construction of the functors, and apply them in examples to obtain representations of the AHA and DAHA, respectively. We obtain representations of the DAHA with five continuous and one discrete parameter: one parameter for each subalgebra, one parameter for each character, the overall quantization parameter q, and finally the integers $N$ and $p$ defining the classical pair; for the AHA we have three continuous parameters: we choose one subalgebra, its character, and we have the overall quantization parameter $\mathrm{q}$. 
The first part of the paper contains the basic constructions, and is organized as follows. In Section 2, we recall the definition of the braid groups and Hecke algebras of type $C^{\vee} C_{n}$. In Section 3, we recall the construction of the braided coordinate algebra, and its relation to reflection equations. In Section 4 , we recall definitions and notation for quasi-triangular Hopf algebras, Noumi co-ideal subalgebras, and their diagrammatic calculus. In Section 5, we construct representations of the affine braid group using the machinery in the preceding sections. In Section 6, we recall the construction of quantum $D$-modules and construct representations of double affine braid group from them.

The remainder of the paper is devoted to connections to the AHA and DAHA coming from quantum groups, and is considerably more technical. In Section 7, we recall the quantum group $\mathcal{U}_{\mathfrak{q}}\left(\mathfrak{g l}_{N}\right)$, the classical symmetric pair $\left(\mathfrak{g l}_{N}, \mathfrak{g l}_{p} \times \mathfrak{g l}_{q}\right)$, and its quantum analog. In Sections 8-9, we show that the constructions of Sections 5 and 6 take values in representations of the AHA and DAHA, respectively, when applied in the context of Section 7. Finally, in Section 10, we compute the quasiclassical limits of our construction and show that they degenerate to those of [EFM].

Acknowledgments. The authors would like to thank Pavel Etingof for his guidance, Ting Xue for helpful discussions, and Stefan Kolb for many helpful comments on our first draft, and for pointing us to Theorem 7.3 . Finally, we thank the anonymous referee for thorough reading and many helpful suggestions and corrections. The work of both authors was supported by NSF grant DMS-0504847.

\section{Double Affine Braid group and Hecke Algebra of type $C^{\vee} C_{n}$}

2.1. The root system $\Phi^{C^{\vee} C_{n}}$ of type $C^{\vee} C_{n}$. Let $\mathcal{E}_{n}=\mathbb{R}^{n}$, with standard basis $\varepsilon_{i}$ and inner product $\left(\varepsilon_{i}, \varepsilon_{j}\right)=\delta_{i j}$. We define the set of roots $\Pi^{C^{\vee} C_{n}}=\left\{ \pm \varepsilon_{i} \pm\right.$ $\left.\varepsilon_{j}\right\}_{i \neq j} \cup\left\{ \pm \varepsilon_{i}\right\} \cup\left\{ \pm 2 \varepsilon_{i}\right\} \subset \mathcal{E}_{n}$. Then $\Phi^{C^{\vee} C_{n}}:=\left(\mathcal{E}_{n}, \Pi^{C^{\vee} C_{n}}\right)$ defines a non-reduced root system. We choose as a set of positive simple roots:

$$
\Pi_{+}^{C^{\vee} C_{n}}=\left\{\alpha_{i}=\varepsilon_{i}-\varepsilon_{i+1}\right\}_{i=1}^{n-1} \cup\left\{\alpha_{n}=\varepsilon_{n}\right\} .
$$

Let $\alpha_{0}$ denote the additional affine positive root. Then $\left\{\alpha_{i}, i=0, \ldots, n\right\}$ form the affine root system of type $C^{\vee} C_{n}$. The corresponding affine Dynkin diagram is

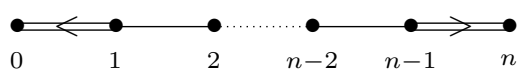

For each $\alpha \in \Pi^{C^{\vee} C_{n}}$, we $s_{\alpha}$ denote the corresponding reflection, and let $s_{i}:=s_{\alpha_{i}}$.

Definition 2.1. The affine Weyl group, $\widehat{\mathcal{W}}_{n}$, of type $C^{\vee} C_{n}$, is the group generated by $s_{0}, \ldots, s_{n}$, with relations $s_{i}^{2}=1$, and the braid relations:

$$
\begin{gathered}
s_{i} s_{j}=s_{j} s_{i},(|i-j|>1), \quad s_{i} s_{i+1} s_{i}=s_{i+1} s_{i} s_{i+1},(i \in\{1, \ldots, n-1\}), \\
s_{0} s_{1} s_{0} s_{1}=s_{1} s_{0} s_{1} s_{0} \quad s_{n-1} s_{n} s_{n-1} s_{n}=s_{n} s_{n-1} s_{n} s_{n-1} .
\end{gathered}
$$

The Weyl group, $\mathcal{W}_{n}$, of type $C^{\vee} C_{n}$, is the subgroup generated by elements $s_{1}, \ldots, s_{n}$. 


\subsection{Double affine braid groups and Hecke algebras in type $C^{\vee} C_{n}$.}

Definition 2.2. The affine braid group, $\widehat{\mathcal{B}}_{n}$ of type $C^{\vee} C_{n}$ is the group generated by $T_{0}, \ldots, T_{n}$, subject to the braid relations:

$$
\begin{array}{r}
T_{i} T_{j}=T_{j} T_{i}, \quad(|i-j|>1), \quad T_{i} T_{i+1} T_{i}=T_{i+1} T_{i} T_{i+1}, \quad(i \in\{1, \ldots, n-1\}), \\
T_{0} T_{1} T_{0} T_{1}=T_{1} T_{0} T_{1} T_{0} \quad T_{n-1} T_{n} T_{n-1} T_{n}=T_{n} T_{n-1} T_{n} T_{n-1}, .
\end{array}
$$

The braid group, $\mathcal{B}_{n}$, is the subgroup generated by $T_{1}, \ldots, T_{n}$.

Definition 2.3. The double affine braid group, $\widetilde{\mathcal{B}}_{n}$, is the group generated by the affine braid group $\widehat{\mathcal{B}}_{n}$ and $K_{0}$, subject to the cross relations:

$$
\begin{aligned}
K_{0} T_{i} & =T_{i} K_{0},(i \in\{2, \ldots, n\}) ; \\
T_{1} K_{0} T_{1} K_{0} & =K_{0} T_{1} K_{0} T_{1} ; \\
T_{0} T_{1}^{-1} K_{0} T_{1} & =T_{1}^{-1} K_{0} T_{1} T_{0} .
\end{aligned}
$$

Remark 2.4. This presentation for the double affine braid group is different from that in [S] and [EGO, and was chosen to allow the most concise constructions for the current work. It is closely related to presentations in [S]. In Section 10.6, it is shown that our presentation agrees with the earlier ones.

For later use, we introduce the following notations:

$$
\begin{aligned}
& T_{(i \cdots j)}:=\left\{\begin{array}{cc}
T_{i} T_{i+1} \cdots T_{j-1}, & j>i>0, \\
T_{i-1} \cdots T_{j+1} T_{j}, & i>j>0, \\
1, & i=j .
\end{array}\right. \\
& P_{i}:=T_{i} \cdots T_{n-1} T_{n} T_{n-1} \cdots T_{i}=T_{(i \cdots n)} T_{n} T_{(n \cdots i)} .
\end{aligned}
$$

Remark 2.5. The group $\widetilde{\mathcal{B}}_{n}$ admits the following geometric description. Let $E$ be an elliptic curve with coordinate $z$, and let

$$
\begin{aligned}
\widetilde{\operatorname{Conf}_{n}}(E):= & \left\{\left(z_{1}, \ldots, z_{n}\right) \in E^{n} \mid z_{i} \neq \pm z_{j}, \text { for } i \neq j, \text { and } z_{i} \neq-z_{i} \cdot\right\} \\
& \operatorname{Conf}_{n}(E):=\widetilde{\operatorname{Conf}_{n}}(E) /\left((\mathbb{Z} / 2 \mathbb{Z})^{n} \rtimes S_{n}\right),
\end{aligned}
$$

where each $\mathbb{Z} / 2 \mathbb{Z}$ replaces $z_{i}$ with $-z_{i}$, and $S_{n}$ permutes the factors. Then one can check that $\pi_{1}\left(\operatorname{Conf}_{n}(E)\right) \cong \widetilde{\mathcal{B}}_{n}$. This is a double affine version of the usual identification $\mathrm{Br}$ of $\mathcal{B}_{n}$ with $\pi_{1}\left(\mathfrak{h}_{\text {reg }} / \mathcal{W}_{n}\right)$, where

$$
\mathfrak{h}_{\text {reg }}:=\left\{\left(z_{1}, \ldots, z_{n}\right) \in \mathbb{C}^{n} \mid z_{i} \neq 0, z_{i} \neq \pm z_{j}, \text { for } i \neq j\right\} .
$$

See Section 10.6 for further discussion.

We fix a field $\mathcal{K}$, and let $v, t, t_{0}, u_{0}, t_{n}, u_{n} \in \mathcal{K} \times$ For an operator $X$ and a parameter $x$, we use the notation $X \sim x$ to mean that $X$ satisfies the Hecke relation $(X-x)\left(X+x^{-1}\right)=0$.

Definition 2.6. The double affine Hecke algebra, $\mathcal{H}_{n}\left(v, t, t_{0}, t_{n}, u_{0}, u_{n}\right)$, of type $C^{\vee} C_{n}$, is the quotient of the group algebra $\mathcal{K}\left[\widetilde{\mathcal{B}}_{n}\right]$ by the Hecke relations:

$$
T_{0} \sim t_{0}, \quad T_{n} \sim t_{n}, \quad K_{0} \sim u_{n}, \quad\left(v K_{0} P_{1} T_{0}\right)^{-1} \sim u_{0}, \quad T_{1}, \ldots, T_{n-1} \sim t .
$$

\footnotetext{
${ }^{2}$ For historical reasons, it is common to replace these parameters formally with their square roots. For simplicity, we have dropped this convention.
} 
The affine Hecke algebra, $\mathcal{H}_{n}\left(t, t_{0}, t_{n}\right)$, of type $C^{\vee} C_{n}$, is the quotient of the group algebra $\mathcal{K}\left[\widehat{\mathcal{B}}_{n}\right]$ by the relations:

$$
T_{0} \sim t_{0}, \quad T_{n} \sim t_{n}, \quad T_{1}, \ldots, T_{n-1} \sim t .
$$

The Hecke algebra, $H_{n}\left(t, t_{n}\right)$, of type $C^{\vee} C_{n}$, is the quotient of the group algebra $\mathcal{K}\left[\mathcal{B}_{n}\right]$ by the relations:

$$
T_{n} \sim t_{n}, \quad T_{1}, \ldots, T_{n-1} \sim t .
$$

Remark 2.7. $\mathcal{H}_{n}\left(t, t_{0}, t_{n}\right)$ and $H_{n}\left(t, t_{n}\right)$ are subalgebras of $\mathcal{H}_{n}\left(v, t, t_{0}, t_{n}, u_{0}, u_{n}\right)$ in the obvious way.

Remark 2.8. There are three variants of the above setup, depending on the choice of $\mathcal{K}$. One may consider: $\mathcal{K}=\mathbb{C}$, and the parameters are numerical, $\mathcal{K}=\mathbb{C}\left(v, t, t_{0}, t_{n}, u_{0}, u_{n}\right)$ and the parameters are indeterminates, $\mathcal{K}=\mathbb{C}((\hbar))$ and the parameters are formal Laurent series. The latter will appear most notably in Section 10 and in that case, we also complete all algebras with respect to $\hbar$.

\section{Characters of the Braided dual and the Reflection Equation}

In this section we recall a categorical construction of a certain quantization of the algebra of functions on an algebraic group, which Majid dubbed the covariantized coordinate algebra, or simply the braided group. For clarity of presentation, we recall some elementary constructions in the theory of tensor categories and phrase our constructions in these terms; of course, we could just as well phrase constructions in terms of generators and relations (see Example 4.5). For details about locally finite tensor categories, see [De1, De2].

Definition 3.1. An abelian category $\mathcal{C}$ is called locally finite if every object $X \in \mathcal{C}$ has finite length, and all Hom spaces are finite dimensional.

Example 3.2. The category of finite dimensional modules over an algebra (possibly infinite dimensional) is a locally finite abelian category, equipped with a functor to vector spaces.

Let $(\mathcal{C}, \otimes, \sigma)$ be a locally finite braided tensor category, and let $\mathcal{C} \otimes \mathcal{C}$ denote its Deligne tensor square. If $\mathcal{C}$ is semisimple, then $\mathcal{C} \otimes \mathcal{C}$ is also, with simples $X \otimes Y$, for $X, Y \in \mathcal{C}$ simple. In any case, we will refer to objects in $\mathcal{C} \otimes \mathcal{C}$ of the form $V \otimes W$ as pure objects: every object in $\mathcal{C} \otimes \mathcal{C}$ is a finite iterated extension of pure objects. $\mathcal{C} \otimes \mathcal{C}$ is also a tensor category with tensor product $\otimes_{2}$, given on pure objects by:

$$
(V \otimes W) \otimes_{2}(X \otimes Y):=(V \otimes X) \otimes(W \otimes Y) .
$$

$\mathcal{C} \otimes \mathcal{C}$ becomes a braided tensor category with braiding $\sigma_{2}:=\sigma \otimes \sigma$. The tensor product on $\mathcal{C}$ gives a functor

$$
T: \mathcal{C} \otimes \mathcal{C} \rightarrow \mathcal{C}, \quad V \otimes W \mapsto V \otimes W .
$$

We can equip $T$ with the structure of a tensor functor by using the braiding $\sigma_{W, X}$ : $\beta: T(V \otimes W) \otimes T(X \otimes Y)=V \otimes W \otimes X \otimes Y \stackrel{\sigma_{W, X}}{\longrightarrow} V \otimes X \otimes W \otimes Y=T\left(V \otimes W \otimes_{2} X \otimes Y\right)$. There is an important ind-algebre $2^{3} \mathbf{A}=\operatorname{CoEnd}(\mathcal{C})$ in $\mathcal{C} \otimes \mathcal{C}$, first constructed by Majid Maj]. As we will use it extensively in what follows, we recall its construction

\footnotetext{
${ }^{3} \mathrm{An}$ ind-object in $\mathcal{C}$ is a direct limit of objects in $\mathcal{C}$, but not, in general, itself an object of $\mathcal{C}$. Rather it is an object in a completion of $\mathcal{C}$ with respect to inductive limits; this distinction is not particularly important for us.
} 
here. To begin, we consider the (very large) ind-object $\widetilde{A}$ in $\mathcal{C} \otimes \mathcal{C}$ :

$$
\widetilde{A}=\bigoplus_{V \in \mathcal{C}} V^{*} \otimes V .
$$

Let $Q \subset \widetilde{A}$ denote the sum over all $V, W$, and $\phi: V \rightarrow W$ of the images in $\widetilde{A}$ of

$$
x_{\phi}:=\phi^{*} \otimes \mathrm{id}_{V}-\mathrm{id}_{W}^{*} \otimes \phi \in \operatorname{Hom}\left(W^{*} \otimes V, V^{*} \otimes V \oplus W^{*} \otimes W\right) .
$$

As an ind-object in $\mathcal{C}$, we define $\mathbf{A}:=\widetilde{A} / Q$. Note that for any object $V \in \mathcal{C}$, we have a canonical map $i_{V}: V^{*} \otimes V \rightarrow \mathbf{A}$. A multiplication $\mu: \mathbf{A} \otimes_{2} \mathbf{A} \rightarrow \mathbf{A}$ is given on each $V^{*} \otimes V, W^{*} \otimes W$ by

$\mu:\left(V^{*} \otimes W^{*}\right) \otimes(V \otimes W) \stackrel{\sigma_{V^{*}, W^{*}} \otimes \text { id }}{\longrightarrow}\left(W^{*} \otimes V^{*}\right) \otimes(V \otimes W) \cong(V \otimes W)^{*} \otimes(V \otimes W)$, which makes $\mathbf{A}$ into a unital associative algebra in $\mathcal{C} \otimes \mathcal{C}$ (one uses the braid relations on the first factor). By tensor functoriality, $T(\mathbf{A})$ also becomes a unital associative algebra in $\mathcal{C}$ with multiplication $T(\mu) \circ \beta$. Furthermore, $T(\mathbf{A})$ carries the structure of a coalgebra in $\mathcal{C}$, with comultiplication defined on generators $V^{*} \otimes V$ :

$$
\Delta:=\mathrm{id}_{V}^{*} \otimes \operatorname{coev}_{V} \otimes \mathrm{id}_{V}: V^{*} \otimes V \rightarrow V^{*} \otimes V \otimes V^{*} \otimes V \subset T(\mathbf{A}) \otimes T(\mathbf{A}) .
$$

The counit is defined on generators by the pairing ev $: V^{*} \otimes V \rightarrow \mathbb{1}$. Any object in $\mathcal{C}$ is naturally both a right and left comodule over $T(\mathbf{A})$ via the maps

$$
\begin{aligned}
& \Delta_{V}^{R}:=\operatorname{coev}_{V} \otimes \mathrm{id}: V \rightarrow V \otimes V^{*} \otimes V \subset V \otimes T(\mathbf{A}), \\
& \Delta_{V}^{L}:=\mathrm{id} \otimes \operatorname{coev}{ }_{V}: V \rightarrow V \otimes{ }^{*} V \otimes V \subset T(\mathbf{A}) \otimes V .
\end{aligned}
$$

Finally, we have the antipode map $S: T(\mathbf{A}) \rightarrow T(\mathbf{A})$ defined on generators by

$$
\left.S\right|_{V^{*} \otimes V}:=\left(u_{V} \otimes \mathrm{id}\right) \circ \sigma_{V^{*}, V}: V^{*} \otimes V \rightarrow V^{* *} \otimes V^{*},
$$

where $u_{V}: V \rightarrow V^{* *}$ is the Drinfeld element (see, e.g. KlSch, p. 247). Together these maps make $T(\mathbf{A})$ into a braided Hopf algebra in $\mathcal{C}$, as defined by Majid Maj]. Note that $\Delta^{L}=\sigma_{V, \mathbf{A}} \circ(\mathrm{id} \otimes S) \circ \Delta^{R}$.

Remark 3.3. A more concise description of $\mathbf{A}$ may be given in the language of module categories. For a $\mathcal{C}$-module category $\mathcal{M}$, and $M, N \in \mathcal{M}$, we let $\underline{\operatorname{Hom}}(M, N) \in \mathcal{C}$ denote the representing object for the functor $\operatorname{Hom}_{\mathcal{M}}(\bullet \otimes M, N)$ (called the inner Homs from $M$ to $N)$. When $M=N$, $\underline{\operatorname{Hom}}(M, M)$ has a natural algebra structure (see $[\mathrm{EO}$ for details). Any tensor category $\mathcal{C}$ has the structure of a $\mathcal{C} \otimes \mathcal{C}^{\otimes-o p}$ module-category, given by $(X \otimes Y) \otimes M:=X \otimes M \otimes Y$. Thus we have an algebra $\mathbf{A}^{\prime}:=\underline{\operatorname{Hom}}(\mathbb{1}, \mathbb{1}) \in \mathcal{C} \otimes \mathcal{C}^{\otimes-o p} ; \mathbf{A}^{\prime}$ represents the functor taking $X \otimes Y$ to the co-invariants of $X \otimes Y$. Finally $\mathbf{A}$ is the $\mathcal{C} \otimes \mathcal{C}$ algebra equivalent to $\mathbf{A}^{\prime}$ via the functor id $\nabla \sigma: \mathcal{C} \otimes \mathcal{C} \rightarrow \mathcal{C} \otimes \mathcal{C}^{\otimes-o p}$. We will not use this construction of A in later sections, but rather its explicit presentation in terms of the relations of equation (4).

Key to applications in Lie theory and quantum groups is the observation that when $\mathcal{C}$ is semi-simple, $\mathbf{A}$ admits the following Peter-Weyl decomposition:

Proposition 3.4. Suppose that $\mathcal{C}$ is semi-simple. Then we have:

$$
\mathbf{A} \cong \bigoplus_{V \text { simple }} V^{*} \otimes V,
$$

where the sum counts each isomorphism class of simple objects exactly once. 
Proof. Apply the relations in equation (4) to isomorphisms $\phi: V \rightarrow W$, to reduce the sum to isomorphism classes of objects $V$. Apply equation (4) to the projections and inclusions of simple components, to further reduce the sum to the simple objects $V$.

\section{Quasi-triangular Hopf algebras}

For the rest of the paper, we work under the assumption that $\mathcal{C}$ is a locally finite braided tensor subcategory of the category of finite dimensional complex representations of a quasi-triangular Hopf algebra $\mathbf{U}$. We denote by $F$ the corresponding tensor functor to vector spaces. For any $\mathbf{U}$-module $V \in \mathcal{C}$, we denote the action by $\rho_{V}: \mathbf{U} \rightarrow \operatorname{End}_{\mathbb{C}}(V)$.

4.1. The universal R-matrix and L-operators. Recall (see, e.g. KlSch for details) that a quasi-triangular Hopf algebra is a Hopf algebra $\mathbf{U}$, with an invertible

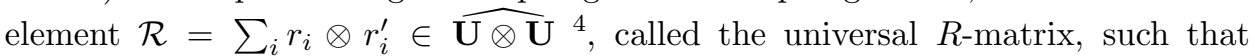
$\Delta^{\mathrm{cop}}(u)=\mathcal{R} \Delta(u) \mathcal{R}^{-1}$, for all $u \in \mathbf{U}$, and

$$
(\Delta \otimes \mathrm{id})(\mathcal{R})=\mathcal{R}_{13} \mathcal{R}_{23}, \quad(\mathrm{id} \otimes \Delta)(\mathcal{R})=\mathcal{R}_{13} \mathcal{R}_{12},
$$

where $\mathcal{R}_{12}=\sum_{i} r_{i} \otimes r_{i}^{\prime} \otimes 1, \mathcal{R}_{13}=\sum_{i} r_{i} \otimes 1 \otimes r_{i}^{\prime}$, and $\mathcal{R}_{23}=\sum_{i} 1 \otimes r_{i} \otimes r_{i}^{\prime}$.

The braiding in $\mathcal{C}$ is given by

$$
\sigma_{V, W}=\tau_{V, W} \circ R_{V, W}: V \otimes W \stackrel{\cong}{\rightrightarrows} W \otimes V,
$$

for any $V, W \in \mathcal{C}$. Here $R_{V, W}:=\rho_{V} \otimes \rho_{W}(\mathcal{R}), \tau_{V, W}$ is the flip operator $V \otimes W \rightarrow$ $W \otimes V, v \otimes w \mapsto w \otimes v$. We will suppress " $\otimes$ id" from morphisms on tensor products when it is clear from context (e.g. $\sigma_{V, W}:=\mathrm{id} \otimes \sigma_{V, W}: \bullet \otimes V \otimes W \rightarrow \bullet \otimes W \otimes V$ ).

Remark 4.1. Usually $\mathcal{R}$ is assumed to lie in $\mathbf{U} \otimes \mathbf{U}$ rather than its completion. However many examples - in particular those coming from quantum groups - fall into this more general context, so we adopt this definition. One could alternatively work with comodules over co-quasitriangular Hopf algebas, but we prefer the present, equivalent, formalism.

For any $\mathbf{U}$-module $V$, we define the " $L$-operators":

$$
\begin{array}{ll}
L_{V}^{+}=\left(\mathrm{id} \otimes \rho_{V}\right)(\mathcal{R}) & \in \mathbf{U} \otimes \operatorname{End}_{\mathbb{C}}(V), \\
L_{V}^{-}=\left(\rho_{V} \otimes \mathrm{id}\right)\left(\mathcal{R}^{-1}\right) & \in \operatorname{End}_{\mathbb{C}}(V) \otimes \mathbf{U} .
\end{array}
$$

For a basis of $V,\left\{e_{i}\right\}$, we define elements $l_{i j}^{V \pm} \in \mathbf{U}$ by

$$
L_{V}^{+}\left(1 \otimes e_{j}\right)=\sum_{i} l_{i j}^{V+} \otimes e_{i}, \quad \text { and } \quad L_{V}^{-}\left(e_{j} \otimes 1\right)=\sum_{i} e_{i} \otimes l_{i j}^{V-} .
$$

We have:

$$
\Delta\left(l_{i j}^{V \pm}\right)=\sum_{k} l_{i k}^{V \pm} \otimes l_{k j}^{V \pm} .
$$

\footnotetext{
${ }^{4}$ For an algebra $A$, let $\hat{A}$ denote its profinite completion, i.e. the completion in the topology in which a basis of neighborhoods of zero is formed by the annihilators of finite dimensional modules. In other words, $\sum_{k} a_{k} \in \hat{A}$ if, and only if, for all $V \in A$-mod finite dimensional, $a_{k} V=0$, for $k \gg 0$.
} 
4.2. The CoEnd algebra A. A fiber functor on $\mathcal{C} \otimes \mathcal{C}$ is defined by $F_{2}:=F \circ T$ : $\mathcal{C} \otimes \mathcal{C} \rightarrow$ Vect. Now let $\mathbf{A}=\operatorname{CoEnd}(\mathcal{C})$ be the ind-algebra in $\mathcal{C}$ defined in Section 3 Then $F_{2}(\mathbf{A})$ becomes an algebra in the usual sense (i.e. in the category of vector spaces), by tensor functoriality.

Remark 4.2. In this case, it is well known that $F_{2}(\mathbf{A})$ is isomorphic as a coalgebra to the restricted dual $\mathbf{U}^{\circ}$ of $\mathbf{U}$, and that the product in $F_{2}(\mathbf{A})$ is twisted from that of $\mathbf{U}^{\circ}$ by a certain cocycle built from the braiding, hence the name "braided dual".

For any $V \in \mathcal{C}$, recall the comodule maps $\Delta_{V}^{R}, \Delta_{V}^{L}$ defined in equations (5), 6). Fixing a basis of $V$, we can write them as matrices, with coefficients in $F_{2}(\mathbf{A})$ :

$$
\Delta_{V}^{R}=\sum_{i, j=1}^{\operatorname{dim} V} E_{i}^{j} \otimes a_{j}^{i}(V), \quad \Delta_{V}^{L}=\sum_{i, j=1}^{\operatorname{dim} V} \tilde{a}_{j}^{i}(V) \otimes E_{i}^{j} .
$$

Here $E_{i}^{j}$ is the matrix $E_{i}^{j} v_{k}=\delta_{j k} v_{i}$. Now suppose $V, W \in \mathcal{C}$ with choosen basis. Define

$$
\begin{aligned}
C_{V}^{R} & :=\sum_{i, j=1}^{\operatorname{dim} V} E_{i}^{j} \otimes \operatorname{id} \otimes a_{j}^{i}(V) \in \operatorname{End}_{\mathbb{C}}(V) \otimes \operatorname{End}_{\mathbb{C}}(W) \otimes T(\mathbf{A}), \\
C_{W}^{R} & :=\sum_{i, j=1}^{\operatorname{dim} W} \mathrm{id} \otimes E_{k}^{l} \otimes a_{l}^{k}(W) \in \operatorname{End}_{\mathbb{C}}(V) \otimes \operatorname{End}_{\mathbb{C}}(W) \otimes T(\mathbf{A}) .
\end{aligned}
$$

Similarly, we have operators $C_{V}^{L}, C_{W}^{L}$ defined using $\Delta_{V}^{L}, \Delta_{W}^{L}$ instead.

Theorem 4.3 ([Maj], DKM]. See [J], Proposition 2.14 for a short proof.). For any $V, W \in \mathcal{C}$, the generators $V^{*} \otimes V$ and $W^{*} \otimes W$ in $F_{2}(\mathbf{A})$ satisfy the relations of the reflection equation algebra:

$$
\begin{gathered}
\sigma_{W, V} C_{V}^{R} \sigma_{V, W} C_{W}^{R}=C_{W}^{R} \sigma_{W, V} C_{V}^{R} \sigma_{V, W}, \\
\sigma_{W, V} C_{W}^{L} \sigma_{V, W} C_{V}^{L}=C_{V}^{L} \sigma_{W, V} C_{W}^{L} \sigma_{V, W} .
\end{gathered}
$$

Example 4.4. If we take $\mathcal{C}$ to be the symmetric category of finite dimensional $\mathcal{U}(\mathfrak{g})$-modules, then the resulting algebra $F_{2}(\mathbf{A})$ is the coordinate algebra $\mathcal{O}(G)$ for the connected, simply connected algebraic group with Lie algebra $\mathfrak{g}$.

Example 4.5. If we instead take $\mathcal{C}$ to be the category of finite dimensional, type $\mathrm{I} \mathcal{U}_{\mathrm{q}}\left(\mathfrak{g l}_{N}\right)$-modules (see Section 7.1), the resulting algebra $F_{2}(\mathbf{A})$ is Majid's covariantized coordinate algebra. $F_{2}(\mathbf{A})$ is twist equivalent (though not isomorphic) to the usual dual quantum group $\mathcal{O}_{\mathrm{q}}(G)$, and has been suggested ([Maj], DM1]) as a preferable replacement for $\mathcal{O}_{\mathrm{q}}(G)$ in the context of braided geometry, as it is constructed to be covariant for the coadjoint action of $\mathcal{U}_{\mathrm{q}}(\mathfrak{g})$.

We can write a presentation of $F_{2}(\mathbf{A})$ explicitly as follows. It is well-known that in this case, $\mathcal{C}$ is generated as a tensor category by the defining representation $\mathbb{C}^{N}$ with highest weight $(1,0, \ldots, 0)$, together with the dual of the determinant representation $\Lambda_{\mathrm{q}}^{N}\left(\mathbb{C}^{N}\right)$. It follows immediately that $F_{2}(\mathbf{A})$ is generated as an algebra by the elements $a_{f, v}, f \in\left(\mathbb{C}^{N}\right)^{*}, v \in \mathbb{C}^{N}$, subject to the relations 110$)$ with $V=W=\mathbb{C}^{N}$, and the inverse of the central element $\operatorname{det}_{\mathrm{q}}$. Even more explicitly, we can choose the standard basis $\left\{e_{i}\right\}$ of weight vectors for $V_{0}$, and its dual basis 
$\left\{e^{i}\right\}$ for $V_{0}^{*}$, and set $a_{j}^{i}:=a_{e^{i}, e_{j}}$. Then $F_{2}(\mathbf{A})$ is the algebra generated by the $a_{j}^{i}$ and $\operatorname{det}_{\mathrm{q}}^{-1}$, subject to relations:

$$
\sum R_{m s}^{i k} a_{l}^{s} R_{u n}^{l m} a_{v}^{n}=\sum a_{l}^{i} R_{m n}^{l k} a_{s}^{n} R_{u v}^{s m} .
$$

As has been noted in many places, these are precisely the so-called "reflection equations".

4.3. Characters of $F_{2}(\mathbf{A})$. Now suppose that $f: F_{2}(\mathbf{A}) \rightarrow \mathbb{C}$ is a character (homomorphism of algebras). For $V \in \mathcal{C}$, let $J_{V}:=\sum_{i, j} f\left(a_{j}^{i}(V)\right) E_{i}^{j}$, and $J_{V}^{\prime}:=$ $\sum_{i, j} f\left(\tilde{a}_{j}^{i}(V)\right) E_{i}^{j}$. Then we have the following well-known

Proposition 4.6. For all $V, W \in \mathcal{C}$, we have the following relation in $\operatorname{End}_{\mathbb{C}}(V \otimes$ $W)$ :

$$
\begin{aligned}
& \sigma_{W, V} J_{V} \sigma_{V, W} J_{W}=J_{W} \sigma_{W, V} J_{V} \sigma_{V, W} . \\
& \sigma_{W, V} J_{W}^{\prime} \sigma_{V, W} J_{V}^{\prime}=J_{V}^{\prime} \sigma_{W, V} J_{W}^{\prime} \sigma_{V, W} .
\end{aligned}
$$

Proof. Apply $f$ to the equations 10 and (11).

We will refer to equations (13) and (14) as the "right-handed" and "left-handed" reflection equations, respectively.

4.4. Coideal subalgebras associated to characters. The operators $J_{V}$ and $J_{V}^{\prime}$ constructed from $f$ in the previous section are not, in general, realized as morphisms of $\mathbf{U}$-modules. Rather, they are morphisms of $\mathbf{B}_{f}$-modules (resp. $\mathbf{B}_{f}^{\prime}$-modules), for certain coideal subalgebras $\mathbf{B}_{f}, \mathbf{B}_{f}^{\prime} \subset \mathbf{U}$ constructed in [KoSt], which we now recall. Let $\mathbf{B}_{f}$ and $\mathbf{B}_{f}^{\prime}$ denote the subalgebras of $\mathbf{U}$ generated by the sets:

$$
\begin{aligned}
& \Phi_{f}:=\left\{c_{i l}=\sum_{j, k=1}^{N} l_{i j}^{V+}\left(J_{V}\right)_{j k} S\left(l_{k l}^{V-}\right) \mid i, l=1, \ldots N\right\}, \\
& \Phi_{f}^{\prime}:=\left\{c_{i l}^{\prime}=\sum_{j, k=1}^{N} S\left(l_{i j}^{V-}\right)\left(J_{V}^{\prime}\right)_{j k} l_{k l}^{V+} \mid i, l=1, \ldots N\right\},
\end{aligned}
$$

respectively. Here $S$ is the antipode of the Hopf algebra $\mathbf{U}$ and $N=\operatorname{dim} V . \mathbf{B}_{f}$ and $\mathbf{B}_{f}^{\prime}$ are independent on the choice of basis, and it follows from $(9)$ that they form left and right coideal subalgebras, respectively:

$$
\Delta\left(\mathbf{B}_{f}\right) \subset \mathbf{U} \otimes \mathbf{B}_{f}, \quad \Delta\left(\mathbf{B}_{f}^{\prime}\right) \subset \mathbf{B}_{f}^{\prime} \otimes \mathbf{U} .
$$

Proposition 4.7. The operator $J_{V} \in \operatorname{End}_{\mathbb{C}}(V)$ is $\mathbf{B}_{f}$-linear: $J_{V}(x v)=x J_{V}(v)$ for all $v \in V$ and $x \in \mathbf{B}_{f}$. The operator $J_{V}^{\prime} \in \operatorname{End}_{\mathbb{C}}(V)$ is $\mathbf{B}_{f}^{\prime}$-linear: $J_{V}^{\prime}(x v)=x J_{V}^{\prime}(v)$ for all $v \in V$ and $x \in \mathbf{B}_{f}^{\prime}$.

Proof. Similar proofs have appeared in many sources, e.g. [KoSt], [DS], [NS]; we include a proof here for the reader's convenience. We prove the statement for $J_{V}$; the statement for $J_{V}^{\prime}$ is similar. To show that $J_{V}$ commutes with all the $\rho_{V}\left(c_{i l}\right)$ is equivalent to showing that $\left(\mathrm{id} \otimes J_{V_{2}}\right)$ commutes with $x=\sum E_{i}^{l} \otimes \rho_{V}\left(c_{i l}\right) \in$ $\operatorname{End}_{\mathbb{C}}\left(V_{1} \otimes V_{2}\right)$, where $V_{1}=V_{2}=V$. We observe that

$$
x=\sum E_{i}^{l} \otimes \rho_{V}\left(l_{i j}^{V+}\left(J_{V}\right)_{j k} S\left(l_{k l}^{V-}\right)\right)=\sigma_{V_{2}, V_{1}} J_{V_{1}} \sigma_{V_{1}, V_{2}},
$$

so that the claim reduces to the right handed reflection equation. 
Remark 4.8. The proof of Proposition 4.7 relies on the observation that the matrix coefficients of $\sigma_{V_{2}, V_{1}} J_{V_{1}} \sigma_{V_{1}, V_{2}}$ are precisely the generators of $\mathbf{B}_{f}$. The same observation provides the key steps in Lemmas 6.9 and 9.4 .

4.5. $J_{V}$-decorated Tangle Diagrams in $\mathcal{C} \otimes \mathcal{C}$. Morphisms in a braided tensor category may be conveniently manipulated using tangle diagram notation (see, e.g. $[\mathrm{K}$, Chapter XIV). It will be necessary to extend the tangle diagram notation in two ways: first, we consider morphisms in the Deligne tensor product $\mathcal{C} \otimes \mathcal{C}$; second, we admit morphisms $J_{V}$ and $J_{V}^{\prime}$ which are not morphisms in $\mathcal{C}$ but rather in the $\mathcal{C}$-module categories of representations of the coideal subalgebras $\mathbf{B}_{f}$ and $\mathbf{B}_{f}^{\prime}$ from Section 4.4 .

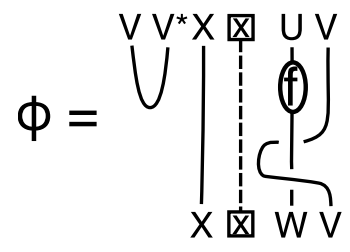

Figure 1. The tangle diagram for the morphism $\phi$ of equation (17).

To depict an object of $\mathcal{C} \otimes \mathcal{C}$, we draw the objects alongside one another, separated by the $₫$ symbol. For a morphism $f \otimes g$ in $\mathcal{C} \otimes \mathcal{C}$, we draw the corresponding tangle diagrams alongside one another, joining the $\nabla$ symbols with a dotted line. We follow the convention from $[\mathrm{K}$ that morphisms move up the page. For example, for $f \in \operatorname{Hom}(W, U)$, Figure 1 depicts the morphism:

$$
\phi=\left(\operatorname{coev}_{V} \otimes \operatorname{id}_{X}\right) \otimes\left(\left(f \otimes \operatorname{id}_{V}\right) \circ \sigma_{W, V}^{-1} \circ \sigma_{V, W}^{-1}\right) .
$$

The linear maps $J_{V}$ and $J_{V}^{\prime}$ do not commute with the braiding in the ordinary way, but may instead be manipulated in a tangle diagram by applying equations (13) and (14), as depicted in Figure 2.

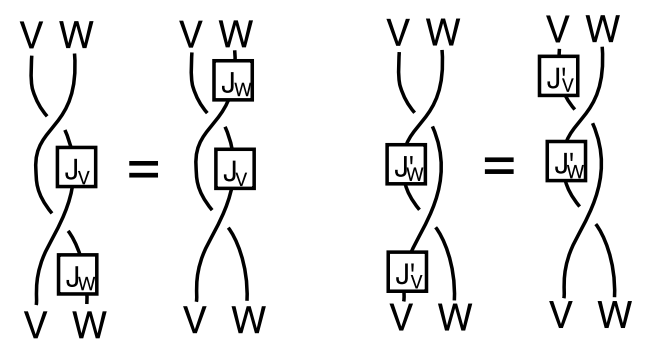

Figure 2. Equality of $J$-decorated tangle diagrams representing equations 13 and 14, respectively. 
5. Some New RePresentations of the AFFine Braid Group of TyPe $C^{\vee} C_{n}$

Let $\mathcal{C}, F$, and $f$ be as in Section 4 . For any objects $M, V_{1}, \ldots, V_{n} \in \mathcal{C}$, consider the vector space 5 .

$$
F_{V_{1}, \ldots, V_{n}}^{f}(M):=M \otimes V_{1} \otimes \cdots \otimes V_{n} .
$$

For simplicity we will take $V_{1}=\cdots=V_{n}=V$ (though it is still convenient to retain the indices), and in this case abbreviate $F_{V, n}^{f}:=F_{V_{1}, \ldots, V_{n}}^{f}$. Our goal in this section is to construct an action of $\widehat{\mathcal{B}}_{n}$ on $F_{n, V}^{f}(M)$. Recall that the character $f$ determines a map $J_{V_{i}}: V_{i} \rightarrow V_{i}$, for each $i$.

In the following construction, we make frequent use of the maps $J_{V}$. As was mentioned in Section 4.5, the only flexibility in moving the morphisms $J_{V}$ about a tangle comes from the reflection equation for $J_{V}$, and so we make repeated use of that identity throughout. We will use the abbreviation QYBE (quantum YangBaxter equation) to refer to relations of undecorated tangle diagrams.

5.1. The action of $\mathcal{B}_{n}$. Let $T_{i}=\sigma_{V_{i}, V_{i+1}}$, for $i=1, \ldots, n-1$. Then it is well known that the $T_{i}$ 's satisfy the braid relations (1). Now let $T_{n}=J_{V_{n}}=$ $\operatorname{id}_{M} \otimes \mathrm{id}^{\otimes(n-1)} \otimes J_{V}$. Then the required relation

$$
T_{n} T_{n-1} T_{n} T_{n-1}=T_{n-1} T_{n} T_{n-1} T_{n}
$$

is equivalent to the right-handed reflection equation for $J_{V_{n}}$. Thus the above construction gives an action of $\mathcal{B}_{n}$ on $F_{n, V}^{f}(M)$. Related constructions have appeared in [KoSt, $\mathrm{tD}$, tDHO], under the name "universal cylinder forms".

5.2. The action of $T_{0}$. We let

$$
T_{0}=P_{1}^{-1}\left(\sigma_{V_{1}, M} \circ \sigma_{M, V_{1}}\right)^{-1}
$$

See Figure 3 for the tangle diagram associated to $T_{0}$. It is straightforward to verify that $T_{i} T_{0}=T_{0} T_{i}$ for $i \geq 2$. We check $T_{1} T_{0} T_{1} T_{0}=T_{0} T_{1} T_{0} T_{1}$ in Figure 4

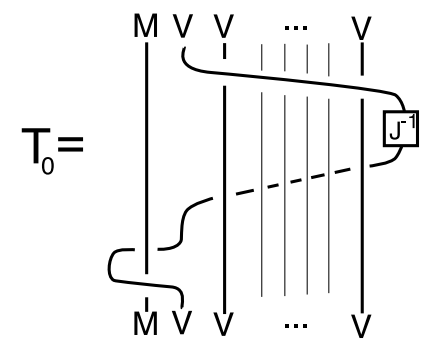

Figure 3 . The morhpism $T_{0}$

We have proven the following:

Theorem 5.1. The operators $T_{0}, \ldots T_{n}$ define a representation of $\widehat{\mathcal{B}}_{n}$ on $F_{n, V}^{f}(M)$. Thus we have a functor:

$$
F_{n, V}^{f}: \mathcal{C} \rightarrow \widehat{\mathcal{B}}_{n}-\bmod , \quad M \mapsto F_{n, V}^{f}(M) .
$$

\footnotetext{
${ }^{5}$ While $f$ does not affect the underlying vector space, it impacts the functor constructed in Theorem 5.1 and so we introduce the notation here.
} 


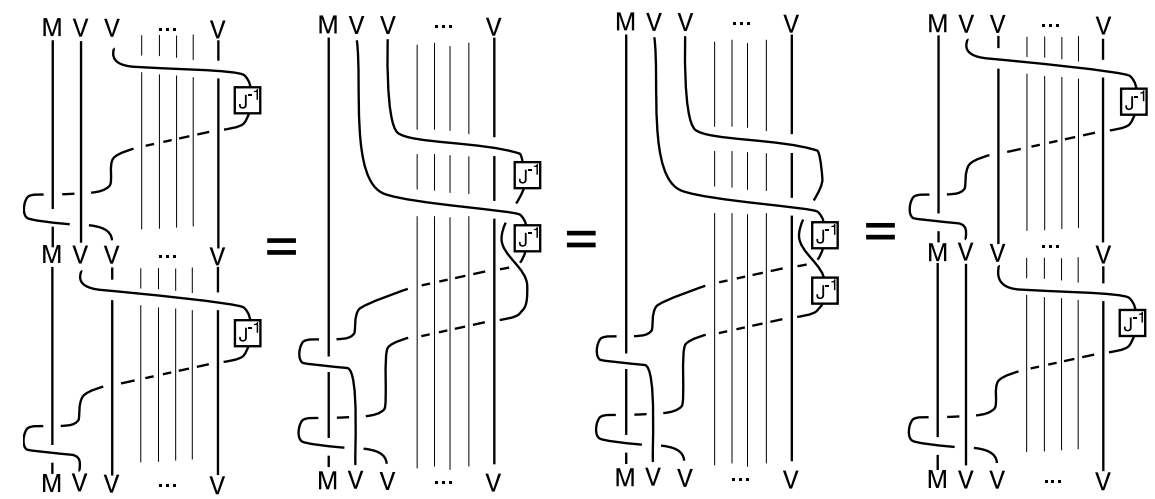

FiguRE 4. Proof of relation $T_{1} T_{0} T_{1} T_{0}=T_{0} T_{1} T_{0} T_{1}$. The first and third equalities use only QYBE, while the second uses the reflection equation for $J$.

Remark 5.2. The pure (double, affine) braid group on $n$ strands is the kernel of the natural projection from the (double, affine) braid group to the symmetric group $\mathcal{S}_{n}$. It is clear from the proof that Theorem 5.1 extends more generally to the pure affine braid group on $n$ strands, if we drop the assumption that all $V_{i}$ are equal. Alternatively, given $V_{1}, \ldots, V_{n}$ possibly distinct, we can construct a similar action of the full affine braid group on the sum

$$
\widetilde{F}_{V_{1}, \ldots V_{n}}^{f}:=\bigoplus_{\sigma \in \mathcal{S}_{n}} M \otimes V_{\sigma(1)} \otimes \ldots \otimes V_{\sigma(n)} .
$$

The same remark applies to Theorem 6.10

\section{Some new Representations of the Double affine Braid group of TYPE $C^{\vee} C_{n}$}

6.1. Quantum $D$-modules. Let $\mathbf{U}$ be a quasi-triangular Hopf algebra, and $\mathcal{C}$ be a locally finite braided tensor subcategory of $\mathbf{U}$-mod, as in Section 4 . The algebra $D_{\mathbf{U}}$ of quantum differential operator ${ }^{6}$ is a Hopf algebra analog of the algebra of differential operators on the algebraic group $G$ with Lie algebra $\mathfrak{g}$ : when $\mathbf{U}=\mathcal{U}(\mathfrak{g})$, we have $D_{\mathbf{U}}=D(G)$. In this section, we recall the definition of $D_{\mathbf{U}}$, and some constructions from [VV] involving it. We have followed their notation as closely as possible, though there are a few differences (in particular, see Remark 6.5).

Let $\mathbf{A}$ be the braided dual algebra defined in Section 3 and let $\widetilde{\mathbf{A}}=(F \otimes F)(\mathbf{A})$. That is, we regard $\mathbf{A}$ as an algebra in vector spaces, where it is equipped with a $(\mathbf{U} \otimes$ $\mathbf{U}$ )-action. Note that $\widetilde{\mathbf{A}}$ is not isomorphic to $F_{2}(\mathbf{A})$ considered previously (although it is obviously twist equivalent). $\widetilde{\mathbf{A}}$ is also a twist-equivalent to a subalgebra of $\mathbf{U}^{*}$. Namely, each $f \otimes v \in \widetilde{\mathbf{A}}$, gives a linear functional $a_{f, v}: \mathcal{U} \rightarrow \mathbb{C}$ by $a_{f, v}(u)=f(u v)$. The product in $\widetilde{\mathbf{A}}$ is such that

$$
\left(a_{f, v} a_{g, w}\right)(u)=\sum a_{r_{i}^{\prime} g \otimes r_{i} f, v \otimes w}(u)=\sum_{i} a_{r_{i} f, v}\left(u_{(1)}\right) a_{r_{i}^{\prime} g, w}\left(u_{(2)}\right) .
$$

\footnotetext{
${ }^{6} \mathbf{A}$ and thus $D_{\mathbf{U}}$ depend on the choice of $\mathcal{C}$, but we will suppress this in the notation
} 
We use " $\triangleright$ " to denote the left adjoint action of $\mathbf{U}$ on itself: for $x, y \in \mathbf{U}, y \triangleright x:=$ $y_{(1)} x S\left(y_{(2)}\right)$, where $\Delta(x)=x_{(1)} \otimes x_{(2)}$ is Sweedler's implicit sum notation for the coproduct. As there is no risk of confusion, we use the same symbol to denote the action of $\mathbf{U} \otimes \mathbf{U}$ on $\widetilde{\mathbf{A}}$ : for $x, y \in \mathbf{U}$, and $f \otimes v \in \widetilde{\mathbf{A}}$, we let $(x \otimes y) \triangleright(f \otimes v):=x f \otimes y v$. In particular, the coadjoint action of $u \in \mathbf{U}$ on $a \in \widetilde{\mathbf{A}}$ is given by $\Delta(u) \triangleright a$. Recall that for vector spaces $V, W, \tau_{V, W}: V \otimes W \rightarrow W \otimes V$ denotes the tensor flip.

Let $\mathbf{U}^{\prime}$ denote the left coideal subalgebra in $\mathbf{U}$ consisting of elements $x$ which generate a finite dimensional submodule under the adjoint action.

Definition 6.1. The algebra $D_{\mathbf{U}}$ of quantum differential operators has underlying vector space $\widetilde{\mathbf{A}} \otimes \mathbf{U}$; the natural inclusions of $\widetilde{\mathbf{A}} \otimes 1$ and $1 \otimes \mathbf{U}$ are algebra homomorphisms, and the commutation relations are given by the smash product:

$$
(1 \otimes x)(a \otimes 1)=\sum_{i, j}\left(\left(x_{(1)} \otimes 1\right) \triangleright a\right) \otimes x_{(2)}, \text { for } a \in \widetilde{\mathbf{A}}, x \in \mathbf{U} .
$$

We denote by $\partial_{\triangleleft}: \mathbf{U} \rightarrow D_{\mathbf{U}}$ the inclusion into the subalgebra $(1 \otimes \mathbf{U})$. The algebra $\mathbf{U}^{\prime}$ is a locally finite left $\mathbf{U}$-module, and thus a right $\widetilde{\mathbf{A}}$ comodule algebra, via the adjoint action; we have a linear map: $\operatorname{ad}^{*}: \mathbf{U}^{\prime} \rightarrow \mathbf{U}^{\prime} \otimes \mathbf{A}, u \mapsto \sum_{i} u_{i} \otimes g_{i}$. By definition, we have $\sum_{i} g_{i}(v) u_{i}=v_{(1)} u S\left(v_{(2)}\right)$, for all $v \in \mathbf{U}$.

Remark 6.2. For quantum groups defined over formal power series, it is not necessary to distinguish between $\mathbf{U}$ and its locally finite part, as the space of vectors of finite type under the ad $\mathbf{U}$-action is dense in the $\hbar$-adic topology, so for instance the co-adjoint map ad* $: \mathbf{U} \rightarrow \mathbf{U} \otimes \widetilde{\mathbf{A}}$ is automatically well-defined as a formal power series - this is all we need.

Proposition 6.3. [VV], Proposition 1.8.2(c), Remark 1.8.4] We have:

(1) If $\mathbf{U}$ has enough finite-dimensional modules (see, e.g. [J], Definition 2.12, Theorem 2.18), then the algebra $\mathbf{A}$ is a faithful representation for $D_{\mathbf{U}} \cdot(W e$ will make this assumption from now on).

(2) The map $\partial_{\triangleright}: \mathbf{U}^{\prime} \rightarrow D_{\mathbf{U}}$, given by

$$
\partial_{\triangleright}(u):=\sum_{i, j}\left(\left(r_{j} \otimes 1\right) \triangleright g_{i}\right) \partial_{\triangleleft}\left(S^{-1}\left(u_{i} r_{j}^{\prime}\right)\right),
$$

is a homomorphism of algebras.

(3) The algebra $\widetilde{\mathbf{A}}$ is equivariant for the resulting $\mathbf{U} \otimes \mathbf{U}^{\prime}$ action.

(4) The images $\partial_{\triangleleft}(\mathbf{U})$ and $\partial_{\triangleright}\left(\mathbf{U}^{\prime}\right)$ commute in $D_{\mathbf{U}}$, so we have a homomorphism $\partial_{2}=\partial_{\triangleleft} \otimes \partial_{\triangleright}: \mathbf{U} \otimes \mathbf{U}^{\prime} \rightarrow D_{\mathbf{U}}$.

(5) $\partial_{2}$ is a quantum moment map: on generators $V^{*} \otimes V$ of $\mathbf{A}$, the $\mathbf{U} \otimes \mathbf{U}^{\prime}$ action is given by:

$$
\partial_{2}(x \otimes y)(f \otimes v)=x f \otimes y v .
$$

Proof. The proof of 6.3 .1 given in [J] applies as well here. We include proofs of 6.3 2-5 for the reader's convenience, as our conventions differ slightly from VV].

For 6.32 , we claim that $\partial_{\triangleright}(u) f=(1 \otimes u) \triangleright f=f(\bullet u)$, for all $f \in \widetilde{\mathbf{A}}$. Here $f(\bullet u)(v):=f(v u)$. This will imply that $\rho_{\widetilde{\mathbf{A}}} \circ \partial_{\triangleright}$, and thus $\partial_{\triangleright}$ by claim 6.31 , is a 
homomorphism. Thus we let $v \in \mathbf{U}$ and compute:

$$
\begin{aligned}
\left(\partial_{\triangleright}(u) f\right)(v) & \left.=\left(\sum_{i, j}\left(r_{j} \otimes 1\right) \triangleright g_{i}\right) \partial_{\triangleleft}\left(S^{-1}\left(u_{i} r_{j}^{\prime}\right)\right) f\right)(v) \\
& =\sum_{i, j}\left(g_{i}\left(S\left(r_{j}\right)-\right) f\left(u_{i} r_{j}^{\prime}-\right)\right)(v) \\
& =\sum_{i, j, k} g_{i}\left(S\left(r_{j}\right) r_{k} v_{(1)}\right) f\left(u_{i} r_{j}^{\prime} r_{k}^{\prime} v_{(2)}\right), \text { by 18) } \\
& =\sum_{k} g_{i}\left(v_{(1)}\right) f\left(u_{i} v_{(2)}\right) \\
& =f\left(v_{(1)} u S\left(v_{(2)}\right) v_{(3)}\right) \\
& =f(v u)
\end{aligned}
$$

as claimed. The proof of 6.3 is clear, because $\widetilde{\mathbf{A}}$ was constructed as an algebra in $\mathcal{C} \otimes \mathcal{C}$. To prove 6.34 , it suffices to consider the $D_{\mathbf{U}}$-module $\widetilde{\mathbf{A}}$, by claim 6.31 . We have

$$
\left(\partial_{\triangleright}\left(u_{1}\right) \partial_{\triangleleft}\left(u_{2}\right) f\right)(v)=f\left(S\left(u_{2}\right) v u_{1}\right)=\left(\partial_{\triangleleft}\left(u_{2}\right) \partial_{\triangleright}\left(u_{1}\right) f\right)(v) .
$$

Claim 6.3.5 follows from the proof of claim 6.3 2 .

Remark 6.4. We will make repeated use of the faithfulness of $\widetilde{\mathbf{A}}$ in coming sections, as in the proofs of 6.32 and 6.34 above. In particular, the proofs of Proposition 6.8 and Theorem 9.1 require us to check certain relations amongst elements in $\operatorname{End}_{\mathbb{C}}(M \otimes U)$, where $M$ is a $D_{\mathbf{U}}$-module, and $U$ is a finite dimensional vector space. Each relation is of the form $\left(\rho_{M} \otimes \mathrm{id}\right)(X)$ for some $X \in D_{\mathbf{U}} \otimes \operatorname{End}_{\mathbb{C}}(U)$, and thus holds for all $D_{\mathbf{U}}$ modules if, and only if, $X$ is already zero. Since $\mathbf{A}$ is faithful, we can verify $X=0$ by evaluating at $M=\mathbf{A}$.

Remark 6.5. The algebra $\widetilde{\mathbf{A}}$ is constructed to be equivariant for a $\mathbf{U} \otimes \mathbf{U}$-action, while the algebra $F_{2}(\mathbf{A})$ is equivariant for the diagonal $\mathbf{U}$-action. In $\mathrm{VV}$ ], there is yet another relative of $\mathbf{A}$, denoted $\mathbf{F}$, which is equivariant for a $\mathbf{U}^{c o-o p} \otimes \mathbf{U}$-action. These algebras are not each isomorphic. However, the smash-product algebras $D_{\mathbf{U}}$ defined from them are isomorphic. See [VV], Proposition 1.4.2 for details.

6.2. Non-degenerate quantum $D$-modules. Classically, a $D(G)$ module is a module over the algebra $\mathcal{U}(\mathfrak{g}) \otimes \mathcal{U}(\mathfrak{g})$ via the inclusions of $\mathcal{U}(\mathfrak{g})$ into $D(G)$ by leftand right-invariant differential operators. The quantum analog of these actions are given by the homomorphism $\partial_{2}: \mathbf{U}^{\prime} \otimes \mathbf{U}^{\prime} \rightarrow D_{\mathbf{U}}$. For $\mathbf{U}=\mathcal{U}(\mathfrak{g})$, we have $\mathbf{U}^{\prime}=\mathbf{U}$, and this recovers the commuting actions entirely; for more general quasi-triangular Hopf algebras $\mathbf{U}$ (including those coming from quantum groups), it can happen that $\mathbf{U}^{\prime} \neq \mathbf{U}$.

We thus introduce the following definitions. We denote by $\widetilde{\mathcal{C}}$ and $\widetilde{\mathcal{D}}$ the categories of $\mathbf{U}$-modules and $\mathbf{U}^{\prime}$-modules, respectively. We denote by Res : $\widetilde{\mathcal{C}} \rightarrow \widetilde{\mathcal{D}}$ the functor of restriction.

Definition 6.6. A non-degenerate $D_{\mathbf{U}}$-module $M$ is an object of $\widetilde{\mathcal{C}} \otimes \widetilde{\mathcal{C}}$, together with the structure of a $D_{\mathbf{U}}$-module on (id $\left.\otimes \operatorname{Res}\right)(M)$, such that the two actions of $\mathbf{U} \otimes \mathbf{U}^{\prime}$ coincide.

Remark 6.7. In other words, we ask for an extension of the action of $\partial_{\triangleright}\left(\mathbf{U}^{\prime}\right)$ to an action of $\mathbf{U}$. For a general quasi-triangular Hopf algebra, it is not completely clear how many $D_{\mathbf{U}}$ modules admit non-degenerate structure. However, see Section 7.3 . 
6.3. Construction of the representations. Let $M$ be a non-degenerate $D_{\mathbf{U}^{-}}$ module. Let $f, g$ be two characters of $\mathbf{A}$, and let $J:=J_{V}$ be the numerical solution to the right-handed reflection equation for $f$, and $J^{\prime}:=J_{V}^{\prime}$ be the numerical solution to the left-handed reflection equation for $g$. Let $\chi: \mathbf{B}_{f} \rightarrow \mathbb{C}$ be a character, and let $\mathbb{1}_{\chi}$ denote the associated one dimensional representation. We regard any nondegenerate $D_{\mathbf{U}}$-module $M$ as a $\mathbf{U} \otimes \mathbf{U}$-module via the homomorphism $\partial_{2}$ of Section 6.1. which we extend to $\mathbf{U} \otimes \mathbf{U}$. We then define (reusing the previous notation):

$$
F_{n, V}^{f, \chi, g}:=\operatorname{Hom}_{\mathbf{B}_{f}}\left(\mathbb{1}_{\chi}, M \otimes_{2}\left(\mathbb{1} \otimes V_{1}\right) \otimes_{2} \cdots \otimes_{2}\left(\mathbb{1} \otimes V_{n}\right)\right) .
$$

In other words, we regard each $V_{i}$ as an object in $\mathcal{C} \otimes \mathcal{D}$, i.e. a $\mathbf{U} \otimes \mathbf{U}^{\prime}$-module with trivial action in the first components. Here $\mathbf{B}_{f}$ acts on the tensor product via the restiction of the homomorphism $\partial_{\triangleright}: \mathbf{B}_{f} \rightarrow D_{\mathbf{U}}$. We let $\widehat{\mathcal{B}}_{n}$ act as before, acting always on the second tensor component (which means it acts by left translation, which are right-invariant quantum vector fields on $M$ ).

We define the following operator

$$
K_{0}:=\mu_{M} \circ \sigma_{1 \otimes V, M} \circ\left(\left(J^{\prime} \otimes 1\right) \circ \operatorname{coev}_{V} \otimes\left(\mathrm{id} \otimes \operatorname{coev}^{*} V\right)\right) \circ \sigma_{1 \otimes V_{1}, M}^{-1},
$$

depicted in the following figure:

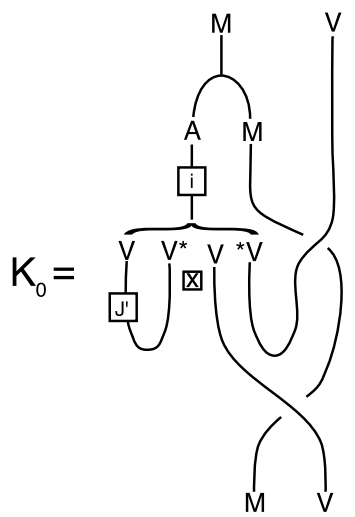

$K_{0}$ is thus constructed from $\mathbf{B}_{f}$-linear ( $\mathbf{U}$-linear, even) morphisms on the second $\bigotimes$-component, and so it automatically preserves spaces of $\mathbf{B}_{f}$-invariants.

Proposition 6.8. We have following identity:

$$
T_{1} K_{0} T_{1} K_{0}=K_{0} T_{1} K_{0} T_{1} \text {, and } K_{0} T_{i}=T_{i} K_{0} \text { for } i \geq 2 .
$$

Proof. The second set of relations is clear because in this case $T_{i}$ and $K_{0}$ act on distinct tensor factors. To show the first relation, we will compute it explicitly in the case $M=\mathbf{A}$, as in Remark 6.4. For this, we can explicitly compute the multiplication $\mu_{M}=\mu_{\mathbf{A}}$ on the generating subspaces $W^{*} \otimes W$ of $\mathbf{A}$, where $K_{0}$ takes the simpler form of Figure 5.

In Figure 6, we prove the relation $T_{1} K_{0} T_{1} K_{0}=K_{0} T_{1} K_{0} T_{1}$.

It remains to show relation (3) in Definition 2.3 .

Lemma 6.9. On the space of $\chi$-invariants, we have the identity

$$
T_{0}^{-1}=\sigma_{V, M} \tilde{J}_{V_{1}} \sigma_{V, M}^{-1} \text {, where } \tilde{J}=\sum E_{i}^{l} \rho_{V}\left(S\left(l_{i j}^{+} \chi\left(c_{j k}\right) S\left(l_{k l}^{-}\right)\right)\right) .
$$




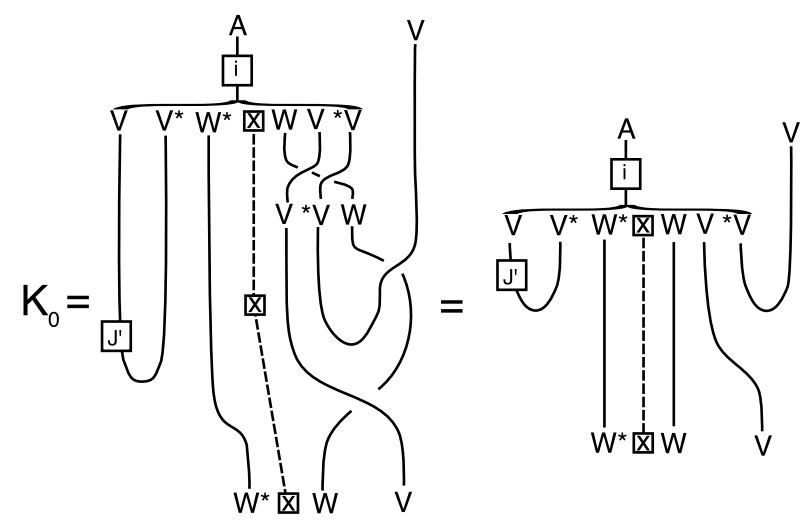

Figure 5. $K_{0}$ acting on the generating subspace $W^{*} \otimes W$ of $\mathbf{A}$.

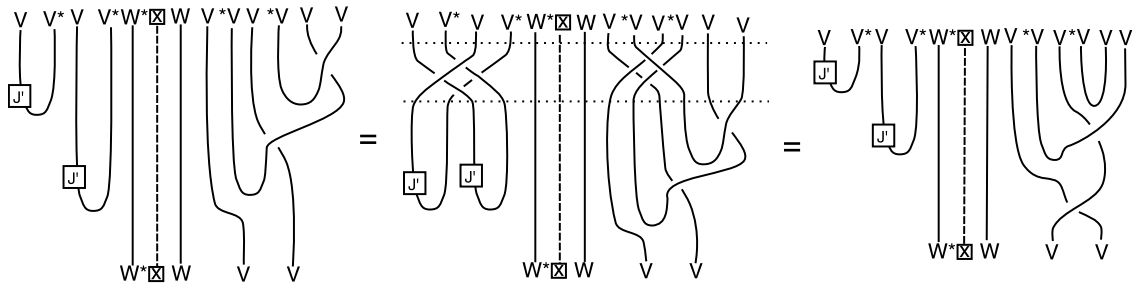

Figure 6. Proof of $T_{1} K_{0} T_{1} K_{0}=K_{0} T_{1} K_{0} T_{1}$. The first equality applies the relations in equation (4) between the dotted lines, noting that the two tangles appearing there are adjoint-inverse to one another. The second equality applies QYBE and the left-handed reflection equation for $J^{\prime}$.

Proof. We compute:

$$
\begin{aligned}
T_{0}^{-1} & =\sigma_{V, M} \sigma_{M, V} T_{(1 \cdots n)} T_{n} T_{(n \cdots 1)} \\
& =\sigma_{V, M} \sigma_{M, V} T_{(1 \cdots n)} T_{n} T_{(n \cdots 1)} \sigma_{V, M} \sigma_{V, M}^{-1} \\
& =\sigma_{V, M}\left(\sum_{i, l}\left(E_{i}^{l}\right)_{V_{1}} \otimes\left(c_{i l}\right)_{M \otimes V_{2} \otimes \cdots V_{n}}\right) \sigma_{V, M}^{-1} \\
& =\sigma_{V, M}\left(\sum E_{i}^{l} \rho_{V}\left(S\left(l_{i j}^{+} \chi\left(c_{j k}\right) S\left(l_{k l}^{-}\right)\right)\right)\right)_{V_{1}} \sigma_{V, M}^{-1},
\end{aligned}
$$

as desired. In the final equality, we have applied the identity

$$
(1 \otimes x)=\left(S\left(x_{(1)}\right) \otimes 1\right)\left(x_{(2)} \otimes x_{(3)}\right)=\left(S\left(x_{(1)}\right) \chi\left(x_{(2)}\right) \otimes 1\right)
$$

to $x=c_{i l}$, using the left coideal property for $\mathbf{B}_{f}$.

The final relation (3) of Definition 2.3 is computed in Figure 7 . We have proven the following: 


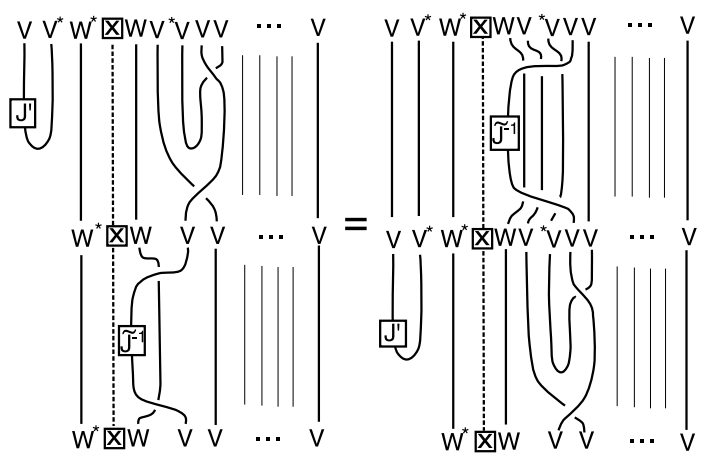

Figure 7. Proof of relation $T_{1}^{-1} K_{0} T_{1} T_{0}=T_{0} T_{1}^{-1} K_{0} T_{1}$. We have applied Lemma 6.9 to simplify the appearance of $T_{0}$ in both sides of the equality. The moves from the left hand side to the right hand side are only QYBE.

Theorem 6.10. The operators $T_{0}, \ldots T_{n}$ and $K_{0}$ define a representation of $\widetilde{\mathcal{B}}_{n}$ on $F_{n, V}^{f, \chi, g}(M)$. We have a functor:

$$
F_{n, V}^{f, \chi, g}: \begin{gathered}
\text { non-degenerate } \\
D_{\mathbf{U}} \text {-modules }
\end{gathered} \longrightarrow \widetilde{\mathcal{B}}_{n} \text {-mod. }
$$

\section{Quantum Groups AND QUANTUM SYMmetric PAIRS}

7.1. The Drinfeld-Jimbo quantum group $\mathcal{U}_{\mathrm{q}}\left(\mathfrak{g l}_{N}\right)$ and its representations. Let $\mathfrak{g}=\mathfrak{g l}(N, \mathbb{C})$ be the Lie algebra of general linear algebraic group $G=\mathrm{GL}(N, \mathbb{C})$. Let $\mathcal{E}_{N}=\mathbb{R}^{N}$, with standard basis $\varepsilon_{i}$ and inner product $\left(\varepsilon_{i}, \varepsilon_{j}\right)=\delta_{i j}$. Let $\Pi_{+}^{A_{N-1}}=\left\{\alpha_{i}=\varepsilon_{i}-\varepsilon_{i+1} \mid i=1, \ldots, N-1\right\}$ be the set of simple roots of $\mathfrak{g}$ and $\Pi^{A_{N-1}}$ be the set of roots. Let $\Lambda$ (resp. $\Lambda^{+}$) be the set of integral (dominant integral) weights for $\mathfrak{g l}_{N}$ :

$$
\begin{gathered}
\Lambda=\left\{m_{1} \varepsilon_{1}+\cdots+m_{N} \varepsilon_{N} \mid m_{i} \in \mathbb{Z}\right\} . \\
\Lambda^{+}=\left\{m_{1} \varepsilon_{1}+\cdots+m_{N} \varepsilon_{N} \mid m_{i} \in \mathbb{Z}, m_{1} \geq \cdots \geq m_{N}\right\} .
\end{gathered}
$$

We let $\omega_{N}:=\varepsilon_{1}+\cdots+\varepsilon_{N}$ denote the fundamental weight corresponding to the determinant representation.

Let $\mathrm{q} \in \mathbb{C}^{\times}$be a nonzero complex number and assume $\mathrm{q}$ is not a root of unity. Set $E_{i}:=E_{\alpha_{i}}$ and $F_{i}:=F_{\alpha_{i}}$ for each simple root. Then the Drinfeld-Jimbo algebra $\mathcal{U}_{\mathrm{q}}(\mathfrak{g})$ is generated by elements $E_{i}, F_{i},(1 \leq i \leq N-1)$, and $K_{j}, K_{j}^{-1}(1 \leq j \leq N)$, with relations:

$$
\begin{gathered}
K_{i} K_{j}-K_{j} K_{i}=0, \quad K_{i} K_{i}^{-1}=K_{i}^{-1} K_{i}=1, \\
K_{i} E_{j} K_{i}^{-1}=\mathrm{q}^{\delta_{i, j}-\delta_{i, j+1}} E_{j}, \quad K_{i} F_{j} K_{i}^{-1}=\mathrm{q}^{-\delta_{i, j}+\delta_{i, j+1}} F_{j}, \\
E_{i} F_{j}-F_{j} E_{i}=\delta_{i, j} \frac{K_{i} K_{i+1}^{-1}-K_{i}^{-1} K_{i+1}}{\mathrm{q}-\mathrm{q}^{-1}}, \\
E_{i} E_{j}-E_{j} E_{i}=0, \quad F_{i} F_{j}-F_{j} F_{i}=0, \quad|i-j| \leq 2, \\
E_{i}^{2} E_{i \pm 1}-\left(\mathrm{q}+\mathrm{q}^{-1}\right) E_{i} E_{i \pm 1} E_{i}+E_{i \pm 1} E_{i}^{2}=0, \\
F_{i}^{2} F_{i \pm 1}-\left(\mathrm{q}+\mathrm{q}^{-1}\right) F_{i} F_{i \pm 1} F_{i}+F_{i \pm 1} F_{i}^{2}=0 .
\end{gathered}
$$


For any $\lambda \in \Lambda$ with $\lambda=\sum_{i} n_{i} \varepsilon_{i}$, we will denote $K^{\lambda}:=K_{1}^{n_{1}} \cdots K_{N}^{n_{N}}$. The Hopf structure on $\mathcal{U}_{\mathrm{q}}(\mathfrak{g})$ is given by:

$$
\begin{gathered}
\Delta\left(K_{i}^{ \pm}\right)=K_{i}^{ \pm} \otimes K_{i}^{ \pm}, \quad \Delta\left(E_{i}\right)=E_{i} \otimes K_{i} K_{i+1}^{-1}+1 \otimes E_{i}, \\
\Delta\left(F_{i}\right)=F_{i} \otimes 1+K_{i}^{-1} K_{i+1} \otimes F_{i}, \quad \epsilon\left(K_{i}\right)=1, \quad \epsilon\left(E_{i}\right)=\epsilon\left(F_{i}\right)=0, \\
S\left(K_{i}\right)=K_{i}^{-1}, \quad S\left(E_{i}\right)=-E_{i} K_{i}^{-1} K_{i+1}, \quad S\left(F_{i}\right)=-K_{i} K_{i+1}^{-1} F_{i} .
\end{gathered}
$$

We will consider the block of type I $\mathcal{U}_{\mathrm{q}}(\mathfrak{g})$-modules, where the generators $K_{i}$ act on a vector $v$ of weight $\mu$ by $\mathrm{q}^{<\varepsilon_{i}, \mu>}$. See [KlSch] for details.

7.2. The vector representation of $\mathcal{U}_{\mathrm{q}}(\mathfrak{g})$. Now let $e_{i}$ be the standard basis for $V=\mathbb{C}^{N}$. The vector representation $\rho_{V}$ of $\mathcal{U}_{\mathrm{q}}(\mathfrak{g})$ on $V=\mathbb{C}^{N}$ is given by:

$$
\begin{gathered}
\rho_{V}\left(K_{i}\right)=\mathrm{q}^{-1} E_{i}^{i}+\sum_{i \neq j} E_{j}^{j}, \quad i=1, \ldots, N, \\
\rho_{V}\left(E_{i}\right)=E_{i+1}^{i}, \quad \rho_{V}\left(F_{i}\right)=E_{i}^{i+1}, \quad i=1, \ldots, N-1 .
\end{gathered}
$$

The $R$-matrix for the vector representation can be expressed explicitly:

$$
R:=\left(\rho_{V} \otimes \rho_{V}\right) \circ \mathcal{R}=\mathrm{q} \sum_{i} E_{i}^{i} \otimes E_{i}^{i}+\sum_{i \neq j} E_{i}^{i} \otimes E_{j}^{j}+\left(\mathrm{q}-\mathrm{q}^{-1}\right) \sum_{i>j} E_{i}^{j} \otimes E_{j}^{i} .
$$

We define $R_{j l}^{i k},\left(R^{-1}\right)_{j l}^{i k} \in \mathbb{C}$, for $i, j, k, l=1, \ldots, N$ by

$$
R\left(e_{i} \otimes e_{j}\right)=\sum_{i, j} R_{i j}^{k l}\left(e_{k} \otimes e_{l}\right), \quad R^{-1}\left(e_{i} \otimes e_{j}\right)=\sum_{i, j}\left(R^{-1}\right)_{i j}^{k l}\left(e_{k} \otimes e_{l}\right) .
$$

We can write the coefficients explicitly as follows:

$$
R_{i j}^{k l}=\left\{\begin{array}{cc}
\mathrm{q}, & i=j=k=l ; \\
1, & i=k \neq j=l ; \\
\mathrm{q}-\mathrm{q}^{-1}, & i=l<j=k ; \\
0, & \text { otherwise }
\end{array} \quad\left(R^{-1}\right)_{i j}^{k l}=\left\{\begin{array}{cc}
\mathrm{q}^{-1}, & i=j=k=l \\
1, & i=k \neq j=l \\
\mathrm{q}^{-1}-\mathrm{q}, & i=l<j=k \\
0, & \text { otherwise }
\end{array}\right.\right.
$$

We will use the notation $L^{ \pm}$and $l_{i j}^{ \pm}$for $L_{V}^{ \pm}$and $l_{i j}^{V, \pm}$, when $V$ is the vector representation. The elements $l_{i j}^{ \pm}$satisfy the following relations:

$$
\begin{gathered}
L_{1}^{ \pm} L_{2}^{ \pm} R=R L_{2}^{ \pm} L_{1}^{ \pm}, \quad L_{1}^{-} L_{2}^{+} R=R L_{2}^{+} L_{1}^{-}, \\
l_{i i}^{+} l_{i i}^{-}=l_{i i}^{-} l_{i i}^{+}=1, \quad i=1, \ldots, N, \\
l_{i j}^{+}=l_{j i}^{-}=0, \quad i>j .
\end{gathered}
$$

Here $L_{ \pm}=\left(l_{i j}^{ \pm}\right)$and $L_{1}^{ \pm}=L^{ \pm} \otimes \mathrm{id}, L_{2}^{ \pm}=\mathrm{id} \otimes L^{ \pm}$which are $N^{2} \times N^{2}$ matrices. In fact, we have the following theorem.

Theorem 7.1 ( See e.g. [KlSch], Ch. 8). The Drinfeld-Jimbo algebra $\mathcal{U}_{\mathrm{q}}(\mathfrak{g})$ is generated by the $l_{i j}^{ \pm}, i, j=1, \ldots, n$, with relations (22), (23), and (24). The antipode $S$, coproduct $\Delta$ and counit $\epsilon$ are given by

$$
S\left(L^{ \pm}\right)=\left(L^{ \pm}\right)^{-1}, \quad \Delta\left(l_{i j}^{ \pm}\right)=\sum_{k} l_{i k}^{ \pm} \otimes l_{k j}^{ \pm}, \quad \text { and } \quad \epsilon\left(l_{i j}^{ \pm}\right)=\delta_{i j} .
$$

By their definition, the elements $l_{i j}^{ \pm}$act on $V=\mathbb{C}^{N}$ via the $R$-matrix; more precisely, we have

$$
\rho_{V}\left(l_{i j}^{+}\right)=\sum_{k, l} R_{l j}^{k i} E_{k}^{l}, \quad \rho_{V}\left(l_{i j}^{-}\right)=\sum_{k, l}\left(R^{-1}\right)_{j l}^{i k} E_{k}^{l} .
$$


7.3. Non-degenerate quantum $D$-modules for $\mathcal{U}_{\mathrm{q}}\left(\mathfrak{g l}_{N}\right)$. In Section 6.2 we have introduced the notion of non-degeneracy for $D_{\mathbf{U}}$-modules. This condition is necessary for technical reasons; however, in this section we show that the restriction is a mild one in the case $\mathbf{U}=\mathcal{U}_{q}\left(\mathfrak{g l}_{N}\right)$ (which we assume in this section).

Proposition 7.2. $\mathbf{U}$ is generated as an algebra by $\mathbf{U}^{\prime}$ and $K_{1}, \ldots, K_{N}$.

Proof. Recall that $x \triangleright y:=x_{(1)} y S\left(x_{(2)}\right)$ denotes the adjoint action of $\mathbf{U}$ on itself. We will use the following theorem due to A. Joseph and G. Letzter. Note that we use slightly different conventions for the $K_{i}$, and that the statement is adapted to account for the central element $K_{\omega_{N}} \in \mathcal{U}_{\mathrm{q}}\left(\mathfrak{g l}_{N}\right)$ ).

Theorem 7.3 (see [JL], Theorem 4.10).

$$
\mathbf{U}^{\prime}=\bigoplus_{\lambda \in-2 \Lambda^{+}+\mathbb{Z} \omega_{N}} \mathcal{U}_{\mathrm{q}}(\mathfrak{g}) \triangleright K^{\lambda} .
$$

Now let $\mathbf{U}^{\prime \prime}$ be the algebra generated by $\mathbf{U}^{\prime}$ and $K_{1}, \ldots, K_{N}$. It is easy to see that $K_{1}^{ \pm 1}, \ldots, K_{N}^{ \pm 1} \in \mathbf{U}^{\prime \prime}$. For $\lambda \in-2 \Lambda^{+}$and $i=1, \ldots, n$, we have:

$$
\begin{aligned}
& E_{i} \triangleright K^{\lambda}=E_{i} K^{\lambda} K_{i+1} K_{i}^{-1}-K^{\lambda} E_{i} K_{i}^{-1} K_{i+1}=\left(1-\mathrm{q}^{\left(\alpha_{i}, \lambda\right)}\right) E_{i} K^{\lambda} K_{i+1} K_{i}^{-1}, \\
& F_{i} \triangleright K^{\lambda}=F_{i} K^{\lambda}-K^{\lambda} F_{i}=\left(1-\mathrm{q}^{-\left(\alpha_{i}, \lambda\right)}\right) F_{i} K^{\alpha} .
\end{aligned}
$$

Thus $E_{i}$ and $F_{i} \in \mathbf{U}^{\prime \prime}$ as well, and so $\mathbf{U}^{\prime \prime}=\mathbf{U}$.

It follows that $\mathbf{U}$ is obtained from $\mathbf{U}^{\prime}$ in a two-step process: first one localizes $\mathbf{U}^{\prime}$ at its denominator set generated by the $K^{-2 \alpha_{i}}$, and then one adjoins a square root $K^{\alpha_{i}}$ of each $K^{2 \alpha_{i}}$.

7.4. The classical symmetric pair and quantum symmetric pair. Let $\mathfrak{g}$ be a reductive Lie algebra with Cartan decomposition $\mathfrak{g}=\mathfrak{n}^{-} \oplus \mathfrak{h} \oplus \mathfrak{n}^{+}$. Suppose we have an involution of $\mathfrak{g}$, denoted by $\theta$. Let $\mathfrak{k}=\mathfrak{g}^{\theta}$ be the fixed Lie subalgebra in $\mathfrak{g}$ under the involution. Then the pair $(\mathfrak{g}, \mathfrak{k})$ is called a (classical) symmetric pair.

Our primary example of a symmetric pair is constructed as follows. Let $\mathfrak{g}=$ $\mathfrak{g l}(N)$ with $N=p+q$. Let $\theta$ be the involutive automorphism of $\mathfrak{g}$ defined by $\theta(u):=J u J$ where

$$
J=\sum_{1 \leq k \leq p} E_{k}^{k}-\sum_{p+1 \leq k \leq N} E_{k}^{k}
$$

The corresponding Lie subalgebra $\mathfrak{k}$ is $\mathfrak{g l}(p) \times \mathfrak{g l}(q)$ and we get the symmetric pair $(\mathfrak{g l}(N), \mathfrak{g l}(p) \times \mathfrak{g l}(q))$. For our purpose, we would like to consider another symmetric pair $\left(\mathfrak{g}, \mathfrak{k}^{\prime}\right)$ as in [DS. The involution $\theta^{\prime}$ of this symmetric pair is given by $\theta^{\prime}(u)=$ $J^{\prime} u J^{\prime}$ with

$$
J^{\prime}=\sum_{1 \leq k \leq p} E_{k}^{N-k+1}+\sum_{1 \leq k \leq p} E_{N-k+1}^{k}-\sum_{p<k<N-p+1} E_{k}^{k} .
$$

It is easy to see that $\mathfrak{k}$ and $\mathfrak{k}^{\prime}$ are conjugate to each other by the matrix $g$ of equation (35).

The theory of quantum symmetric pairs provides an analog of classical symmetric pairs in the setting of quantum groups. It was developed systematically by G. Letzter in a series of papers [L1, L2], with many examples coming from so-called Noumi coideal subalgebras $[\mathrm{N}, \mathrm{NS}, \mathrm{OS}$.

Let $(\mathfrak{g}, \mathfrak{k})$ denote a classical symmetric pair. A quantum symmetric pair associated to $(\mathfrak{g}, \mathfrak{k})$ is a pair $\left(\mathcal{U}_{\mathrm{q}}(\mathfrak{g}), \mathcal{I}\right)$, where $\mathcal{I}$ is a right coideal subalgebra in $\mathcal{U}_{\mathrm{q}}(\mathfrak{g})$, 
such that the quasi-classical limit as $\mathrm{q} \rightarrow 1$ recovers $\mathcal{U}(\mathfrak{k})$. The coideal formalism arises because while $\mathcal{U}(\mathfrak{k})$ is a sub-Hopf algebra of $\mathcal{U}(\mathfrak{g})$, the quantization $\mathcal{I}$ of $\mathcal{U}(\mathfrak{k})$ inside $\mathcal{U}_{\mathrm{q}}(\mathfrak{g})$ is no longer a sub-coalgebra, but only a one-sided coideal.

7.5. The one parameter family of coideal subalgebras. The symmetric pair $\left(\mathfrak{g}, \mathfrak{k}^{\prime}\right)$ can be quantized via the method of characters $f: F_{2}(\mathbf{A}) \rightarrow \mathbb{C}$, where $F_{2}(\mathbf{A})$ is the braided dual of $\mathcal{U}_{\mathrm{q}}\left(\mathfrak{g l}_{N}\right)$. Let $\left\{a_{j}^{i}\right\}$ be the generators of $F_{2}(\mathbf{A})$ which are defined in Section 4.2. Characters for the reflection equation algebra associated to $\mathcal{U}_{\mathrm{q}}\left(\mathfrak{g l}_{N}\right)$ were studied by Donin, Kulish and Mudrov DKM, DM1, DM2, and completely classified in $\mathrm{Mud}$. In $\mathrm{KoSt}$, it was explained that a character $f$ of the reflection equation algebra extends to a character of the braided dual of $\mathcal{U}_{\mathrm{q}}\left(\mathfrak{g l}_{N}\right)$ if, and only if, the matrix $\left(f\left(a_{j}^{i}\right)\right)$ is invertible. Following them (see also [N, OS, DS]), we choos $€^{7} \mathrm{q}^{\sigma} \in \mathbb{C}$, and define an $N \times N$ complex matrix $J^{\sigma}$ :

$$
J^{\sigma}=\sum_{1 \leq k \leq p}\left(\mathrm{q}^{\sigma}-\mathrm{q}^{-\sigma}\right) E_{k}^{k}-\sum_{p<k<N-p+1} \mathrm{q}^{-\sigma} E_{k}^{k}+\sum_{1 \leq k \leq p} E_{k}^{N-k+1}+\sum_{1 \leq k \leq p} E_{N-k+1}^{k} .
$$

Note that $J^{\sigma}$ satisfies a Hecke relation $J^{\sigma} \sim \mathrm{q}^{\sigma}$.

Lemma 7.4 (See e.g. [DS], [DNS, $[\mathrm{Mud}]$ ). The matrix $J^{\sigma}$ is a right-handed numerical solution of the reflection equation

$$
R_{21} J_{1}^{\sigma} R_{12} J_{2}^{\sigma}=J_{2}^{\sigma} R_{21} J_{1}^{\sigma} R_{12},
$$

where $J_{1}^{\sigma}=J^{\sigma} \otimes$ id and $J_{2}^{\sigma}=\mathrm{id} \otimes J^{\sigma}$.

Corollary 7.5. The matrix $\left(J^{\sigma}\right)^{-1}$ is a left-handed numerical solution of the reflection equation.

Proof. By the lemma, $J^{\sigma}$ is a solution of the right handed reflection equation for all $\mathrm{q}^{\sigma} \in \mathbb{C}$. Let us write $R=R(\mathrm{q})$ and $J^{\sigma}=J^{\sigma}(\mathrm{q})$ to emphasize the dependence on q. By inspecting the $R$-matrix for $V \otimes V$, we see that $R(\mathrm{q})^{-1}=R\left(\mathrm{q}^{-1}\right)$. Similarly $J^{\sigma}(\mathrm{q})=J^{-\sigma}\left(\mathrm{q}^{-1}\right)$. Thus, we compute that the left handed reflection equation for $J^{-\sigma}$ at $\mathrm{q}$ is equivalent to the left-handed equation for $\left(J^{\sigma}\right)^{-1}$ at $\mathrm{q}^{-1}$ :

$$
\begin{aligned}
R_{21}(\mathrm{q}) J_{1}^{-\sigma}(\mathrm{q}) R_{12}(\mathrm{q}) J_{2}^{-\sigma}(\mathrm{q}) & =J_{2}^{-\sigma}(\mathrm{q}) R_{21}(\mathrm{q}) J_{1}^{-\sigma}(\mathrm{q}) R_{12}(\mathrm{q}) \\
\Leftrightarrow R_{21}\left(\mathrm{q}^{-1}\right)^{-1} J_{1}^{\sigma}\left(\mathrm{q}^{-1}\right) R_{12}\left(\mathrm{q}^{-1}\right)^{-1} J_{2}^{\sigma}\left(\mathrm{q}^{-1}\right) & =J_{2}^{\sigma}\left(\mathrm{q}^{-1}\right) R_{21}\left(\mathrm{q}^{-1}\right)^{-1} J_{1}^{\sigma}\left(\mathrm{q}^{-1}\right) R_{12}\left(\mathrm{q}^{-1}\right)^{-1}, \\
\Leftrightarrow J_{2}^{\sigma}\left(\mathrm{q}^{-1}\right)^{-1} R_{12}\left(\mathrm{q}^{-1}\right) J_{1}^{\sigma}\left(\mathrm{q}^{-1}\right)^{-1} R_{21}\left(\mathrm{q}^{-1}\right) & =R_{12}\left(\mathrm{q}^{-1}\right) J_{1}^{\sigma}\left(\mathrm{q}^{-1}\right)^{-1} R_{21}\left(\mathrm{q}^{-1}\right) J_{2}^{\sigma}\left(\mathrm{q}^{-1}\right)^{-1}, \\
\Leftrightarrow J_{1}^{\sigma}\left(\mathrm{q}^{-1}\right)^{-1} R_{21}\left(\mathrm{q}^{-1}\right) J_{2}^{\sigma}\left(\mathrm{q}^{-1}\right)^{-1} R_{12}\left(\mathrm{q}^{-1}\right) & =R_{21}\left(\mathrm{q}^{-1}\right) J_{2}^{\sigma}\left(\mathrm{q}^{-1}\right)^{-1} R_{12}\left(\mathrm{q}^{-1}\right) J_{1}^{\sigma}\left(\mathrm{q}^{-1}\right)^{-1} .
\end{aligned}
$$

The first equivalence follows from the preceding paragraph. The second is by inverting both sides of the equation, and the third is by applying the flip $\tau_{12}$. Since the right handed reflection equation is established for $J^{\sigma}(\mathrm{q})$ at all parameters $\mathrm{q}$ and $\mathrm{q}^{\sigma}$, it follows that the left hand reflection equation holds for $J^{\sigma}(\mathrm{q})$ for all $\mathrm{q}$ and $\mathrm{q}^{\sigma}$ as well.

Thus we can define characters $f_{\sigma}: F_{2}(\mathbf{A}) \rightarrow \mathbb{C}, f_{\sigma}\left(a_{i}^{j}\right):=J_{i j}^{\sigma}$, and $g_{\rho}: F_{2}(\mathbf{A}) \rightarrow$ $\mathbb{C}, g_{\rho}\left(\tilde{a}_{i}^{j}\right)=\left(\left(J^{\rho}\right)^{-1}\right)_{i j}$. Note that the corresponding matrices $J_{V}:=\sum f\left(a_{j}^{i}\right) E_{i}^{j}$ and $J_{V}^{\prime}:=\sum g\left(\tilde{a}_{j}^{i}\right) E_{i}^{j}$ for the vector representation $V=\mathbb{C}^{N}$ will be $J^{\sigma}$ and $\left(J^{\rho}\right)^{-1}$

\footnotetext{
${ }^{7}$ In this article $\mathrm{q}^{\sigma}$ denotes a generic complex number, not directly related to $\mathrm{q}$. We keep the old notation for two reasons: first to emphasize the connection with previous papers [DS, $\mathrm{NS}$, DS, and second, because in the formal setting we will take $\sigma \in \mathbb{C}$, and let $\mathrm{q}:=e^{\hbar}$, and $\mathrm{q}^{\sigma}:=e^{\sigma \hbar}$, in order to compute the trigonometric degeneration. We let $\mathrm{q}^{-\sigma}:=\frac{1}{\mathrm{q}^{\sigma}}$.
} 
themselves, since $J^{\sigma}$ and $\left(J^{\rho}\right)^{-1}$ are symmetric. Following section 4.4 we have coideal subalgebras $\mathbf{B}_{\sigma}:=\mathbf{B}_{f_{\sigma}}$ and $\mathbf{B}_{\rho}^{\prime}:=\mathbf{B}_{g_{\rho}}^{\prime}$ associated to any $V \in \mathcal{C}$. 8

In Letzter's framework [L1, L2], it is important that the coideal subalgebras $\mathbf{B}_{\sigma}$ are all isomorphic as abstract algebras (similarly for the $\mathbf{B}_{\rho}^{\prime}$ ). This property was also used in $\mathrm{OS}$ in the case $p=q$, where the authors constructed a single comodule algebra and a family of embeddings into the quantum group. In our case, the isomorphisms between the $\mathbf{B}_{\sigma}$ take an especially simple form in the following propositon:

Proposition 7.6. Let $\mathrm{q}, \mathrm{q}^{\sigma_{1}}, \mathrm{q}^{\sigma_{2}} \in \mathbb{C}$ be generic, and let $\phi: \mathbf{B}_{\sigma_{1}} \rightarrow \mathbf{B}_{\sigma_{2}}$ be defined on generators by $\phi\left(c_{i l}^{(1)}\right)=c_{i l}^{(2)}$, where $c_{i l}^{(k)}$ are the generators 15 for $\mathbf{B}_{\sigma_{k}}$. Then $\phi$ is an isomorphism of algebras.

Proof. Using that $L^{+}$(resp. $L^{-}$) is upper (resp. lower) triangular, that $S\left(l_{i i}^{-}\right)=l_{i i}^{+}$, and that $J^{\sigma}$ is skew-upper triangular and symmetric, we can see by inspection that the matrix of generators $\left(c_{i l}\right)$ has the form:

$$
c_{i l}=\left(\begin{array}{lll}
* & * & X \\
* & * & 0 \\
Y & 0 & 0
\end{array}\right)_{i l},
$$

where the blocks are of size $(p, q-p, p) \times(p, q-p, p)$ (the same as in $\left.J^{\sigma}\right)$. Here, the $*$ 's are some nonzero expressions, $X$ and $Y$ are skew upper triangular, and we have $X_{i, p-i}=Y_{p-i, i}$. This means that each $\mathcal{I}_{\sigma}$ is really generated by the $q^{2}$ entries in the $*^{\prime}$ ed regions, plus the $p^{2}$ entries in $X$ and $Y$, counting the diagonal only once. This gives a system of $p^{2}+q^{2}$ generators, which are subject to (at least) the relations of the reflection equation algebra:

$$
R_{21} c_{1} R_{12} c_{2}=c_{2} R_{21} c_{1} R_{12} \text {. }
$$

It follows that the algebras $\mathbf{B}_{\sigma}$ are spanned by ordered monomials in the $c_{i l}$, though a priori we may expect more relations.

It turns out that there are no other relations, which we can see as follows. It is shown in Section 10.4 that the quasi-classical limits of the elements $c_{i l}$ are the generators of the subalgebra $\mathcal{U}(\mathfrak{k})=\mathcal{U}\left(\mathfrak{k}^{\prime}\right) \subset \mathcal{U}\left(\mathfrak{g l}_{N}\right)$, which itself affords a PBW basis of ordered monomials in its generators. It now follows from the fact that $\mathcal{U}_{\mathrm{q}}(\mathfrak{g})$ is a flat deformation of $\mathcal{U}(\mathfrak{g})$, for q not a root of unity, that the relations 28 provide all the relations on $\mathbf{B}_{\sigma}$. In particular, the relations don't depend at all on $\mathrm{q}^{\sigma}$, so the map $\phi$ is an isomorphism.

Obviously the map $\chi_{\sigma}: c_{i l} \mapsto J_{i l}^{\sigma}$ is a character of $\mathbf{B}_{\sigma}\left(\chi_{\sigma}\right.$ is the restriction of $\epsilon)$. In fact, we see by the previous proposition that each $\mathbf{B}_{\sigma}$ has a two parameter family of characters:

$$
\chi_{\tau}^{\eta}\left(l_{i j}^{+} J_{j k}^{\sigma} S\left(l_{k l}^{-}\right)\right):=\mathrm{q}^{\eta} J_{i l}^{\tau} .
$$

Likewise, each $\mathbf{B}_{\rho}^{\prime}$ has a two parameter family of characters:

$$
\lambda_{\nu}^{\omega}\left(S\left(l_{i j}^{-}\right)\left(J^{\rho}\right)_{j k}^{-1} l_{k l}^{+}\right):=q^{\omega}\left(J^{\nu}\right)_{i l}^{-1} .
$$

In the next two sections, we will use these to construct twisted invariants and twisted quantum $D$-modules.

\footnotetext{
${ }^{8}$ It is also possible to scale the matrices $J^{\sigma}$ by an arbitrary nonzero complex number. Of course, doing so will yield the same algebra.
} 
7.6. q-Harish Chandra modules for $\left(\mathcal{U}_{\mathrm{q}}\left(\mathfrak{g l}_{N}\right), \mathbf{B}_{\sigma}\right)$. In the theory of real and p-adic groups, an important role is played by the so-called Harish-Chandra modules associated to a symmetric pair $(G, K)$. The following definition captures the relevant algebraic properties in the q-deformed setting, and was proposed in [L3], Definition 3.1.

Definition 7.7. The category of q-Harish-Chandra modules for $\left(\mathcal{U}_{\mathrm{q}}\left(\mathfrak{g l}_{N}\right), \mathbf{B}_{\sigma}\right)$ is the full abelian subcategory of $\mathcal{U}_{\mathrm{q}}\left(\mathfrak{g l}_{N}\right)$-modules $M$ such that $\mathbf{B}_{\sigma}$ acts semi-simply on $M$.

Definition 7.8. The category of q-Harish-Chandra $D$-modules for $\left(\mathcal{U}_{\mathrm{q}}\left(\mathfrak{g l}_{N}\right), \mathbf{B}_{\sigma}, \mathbf{B}_{\rho}^{\prime}\right)$ is the full abelian subcategory of non-degenerate $\left.D_{\mathcal{U}_{\mathfrak{q}}\left(\mathfrak{g l}_{N}\right)}\right)^{\text {-modules }}$ $M$ such that $\partial_{2}\left(\mathbf{B}_{\rho}^{\prime} \otimes \mathbf{B}_{\sigma}\right)$ acts semi-simply on $M$.

In either case, we have the "Harish-Chandra part" functor which sends a module to sum of all its q-Harish Chandra submodules; the result is only a $\mathbf{U}^{\prime} \mathbf{B}_{\sigma}$-module (see the discussion in [L3] following Definition 3.1). In the case of non-degenerate $D_{\mathbf{U}}$-modules, the Harish-Chandra part is only a $\mathbf{U}^{\prime} \mathbf{B}_{\rho}^{\prime} \otimes \mathbf{U}^{\prime} \mathbf{B}_{\sigma}$ module, which is preserved by the $\mathbf{A}$ action. This is enough for our purposes.

\section{Representations of the affine Hecke algebras of type $C^{\vee} C_{n}$.}

Let $V=\mathbb{C}^{N}$ be the vector representation for $\mathcal{U}_{\mathrm{q}}(\mathfrak{g})=\mathcal{U}_{\mathrm{q}}\left(\mathfrak{g l}_{N}\right)$. Let $\chi_{\tau}^{\eta}$ be the character of $\mathbf{B}_{\sigma}$ defined in $(29)$, and let $\mathbb{1}_{\tau}^{\eta}$ denote the associated one-dimensional representation. For any $\mathbf{B}_{\sigma}$-module $W$, we denote by $W^{\text {l.f. }}$ the locally finite part of $W$, i.e. the sum of all finite dimensional $\mathbf{B}_{\sigma}$-submodules of $W$. For any $\mathcal{U}_{\mathrm{q}}(\mathfrak{g})$ module $M$, define a vector space

$$
F_{n}^{\sigma, \eta, \tau}(M)=\left(M \otimes V^{\otimes n}\right)^{\mathbf{B}_{\sigma}, \chi_{\tau}^{\eta}}:=\operatorname{Hom}_{\mathbf{B}_{\sigma}}\left(\mathbb{1}_{\tau}^{\eta}, M \otimes V^{\otimes n}\right) .
$$

Above, the $\mathbf{B}_{\sigma}$ action on the tensor product is as in Section 6 . The main result of this section is the following theorem.

Theorem 8.1. $F_{n}^{\sigma, \eta, \tau}$ defines an functor from the category of $\mathcal{U}_{\mathrm{q}}(\mathfrak{g})$-modules to the category of representations of the affine Hecke algebra $\mathcal{H}_{n}\left(t, t_{0}, t_{n}\right)$ with parameters:

$$
t=\mathrm{q}, \quad t_{n}=\mathrm{q}^{\sigma}, \quad t_{0}=\mathrm{q}^{(p-q-\tau)} .
$$

Moreover $F_{n}^{\sigma, \eta, \tau}$ factors through the Harish-Chandra part functor, and is exact on the category of q-Harish-Chandra modules.

The construction is a specialization of Section 5, except that we rescale the operators to have eigenvalues of the form $\lambda,-\lambda^{-1}$. It is clear that the relations we checked in Section 5 are unchanged by rescaling; thus, the only new proofs in this section will be checking the Hecke relations.

For $i=1, \ldots n-1$, we let $T_{i}=\sigma_{V_{i}, V_{i+1}}$, and we let $T_{n}=J_{V_{n}}^{\sigma}$. We let $T_{0}=$ $\alpha P_{1}^{-1}\left(\sigma_{V_{1}, M} \circ \sigma_{M, V_{1}}\right)^{-1}$, where $\alpha=\mathrm{q}^{-N+\eta}$. It follows immediately that $T_{i} \sim \mathrm{q}$, and $T_{n} \sim \mathrm{q}^{\sigma}$.

Proposition 8.2. $T_{0} \sim \mathrm{q}^{p-q-\tau}$. 
Proof. By Lemma 6.9, on the space of $\left(\mathcal{I}_{\sigma}, \chi_{\tau}\right)$-invariants, $T_{0}^{-1}$ has the same minimal polynomial as $\alpha^{-1} \tilde{J}=\mathrm{q}^{N-\eta} \sum E_{i}^{l} \rho\left(S\left(l_{i j}^{+} \chi_{\tau}^{\eta}\left(c_{j k}\right) S\left(l_{k l}^{-}\right)\right)\right)$. Applying the definition of $\chi_{\tau}^{\eta}$, we have:

$$
\begin{aligned}
\alpha^{-1} \tilde{J} & =\mathrm{q}^{N} \sum E_{i}^{l} \rho\left(S\left(l_{i j}^{+} J_{j k}^{\tau} S\left(l_{k l}^{-}\right)\right)\right) \\
& =\mathrm{q}^{N} \sum E_{i}^{l} \rho\left(S^{2}\left(l_{k l}^{-}\right) J_{j k}^{\tau} S\left(l_{i j}^{+}\right)\right) \\
& =\mathrm{q}^{N} \sum E_{i}^{l} \rho\left(u l_{k l}^{-} u^{-1} J_{j k}^{\tau} S\left(l_{i j}^{+}\right)\right),
\end{aligned}
$$

where $u$ is the Drinfeld element such that $S^{2}(x)=u x u^{-1}$ for all $x \in \mathbf{U}$. For the vector representation we have the well-known formuld?

$$
\rho_{V}(u)=\sum_{i=1}^{N} \mathrm{q}^{2 i-2} E_{i}^{i} .
$$

By equations 200 and 21) and direct computation, we have

$$
\begin{aligned}
\alpha^{-1} \tilde{J}=\sum_{i=1}^{p} & \left(\mathrm{q}^{q-p+\tau}-\mathrm{q}^{p-q-\tau}\right) E_{i}^{i}-\sum_{i=p+1}^{N-p} \mathrm{q}^{p-q-\tau} E_{i}^{i} \\
& +\sum_{i=1}^{p} \mathrm{q}^{-N+2 i-1} E_{i}^{N+1-i}+\sum_{i=1}^{p} \mathrm{q}^{N-2 i+1} E_{N+1-i}^{i},
\end{aligned}
$$

which is semisimple, with two eigenvalues: $\lambda_{1}=\mathrm{q}^{q-p+\tau}$ and $\lambda_{2}=-\mathrm{q}^{p-q-\tau}$.

The second part of the theorem follows easily because tensoring is an exact functor, as is $\operatorname{Hom}(\mathbb{1}, \bullet)$, when restricted to the category of q-Harish-Chandramodules.

\section{Representations of the double affine Hecke algebras of type $C^{\vee} C_{n}$}

Let $V=\mathbb{C}^{N}$ denote the vector representation for $\mathbf{U}=\mathcal{U}_{\mathrm{q}}\left(\mathfrak{g l}_{N}\right)$. Let $\chi_{\tau}^{\eta}$ and $\lambda_{\nu}^{\omega}$ be the characters of $\mathbf{B}_{\sigma}$ and $\mathbf{B}_{\rho}^{\prime}$, respectively, defined in equations 29 and (30). We denote the corresponding one dimensional representations $\mathbb{1}_{\tau}^{\eta}:=\mathbb{1}_{\chi_{\tau}^{\eta}}$ and $\mathbb{1}_{\nu}^{\omega}:=\mathbb{1}_{\lambda_{\omega}^{\omega}}$. In this section we prove that a certain rescaling of the action defined in Section 6 induces an action of the double affine Hecke algebra of type $C^{\vee} C_{n}$. Let $M$ be a non-degenerate $D_{\mathbf{U}}$-module, and let

$$
F_{n, \rho, \omega, \nu}^{\sigma, \eta, \tau}(M):=\operatorname{Hom}_{\mathbf{B}_{\rho}^{\prime} \otimes \mathbf{B}_{\sigma}}\left(\mathbb{1}_{\nu}^{\omega} \otimes \mathbb{1}_{\tau}^{\eta}, M \otimes_{2}\left(\mathbb{1} \otimes V_{1}\right) \otimes_{2} \cdots \otimes_{2}\left(\mathbb{1} \otimes V_{n}\right)\right) .
$$

Theorem 9.1. $F_{n, \rho, \omega, \nu}^{\sigma, \eta, \tau}$ defines a functor from the category of non-degenerate $D_{\mathbf{U}}$-modules to the category of representations of the double affine Hecke algebra $\mathcal{H}_{n}\left(v, t, t_{0}, t_{n}, u_{0}, u_{n}\right)$ with parameters:

$$
\begin{gathered}
t=\mathrm{q}, \quad t_{n}=\mathrm{q}^{\sigma}, \quad t_{0}=\mathrm{q}^{(p-q-\tau)}, \\
u_{0}=\mathrm{q}^{\nu}, \quad u_{n}=\mathrm{q}^{-\rho}, \quad v=\mathrm{q}^{\eta-N-\omega} .
\end{gathered}
$$

Moreover $F_{n, \rho, \omega, \nu}^{\sigma, \eta, \tau}$ factors through the $\mathrm{q}$-Harish-Chandra part of $M$, and is an exact functor on the category $\mathbf{q}$-Harish-Chandra $D_{\mathbf{U}}$-modules.

\footnotetext{
${ }^{9}$ up to an immaterial scalar, depending on the normalization of $u$.
} 
We let $T_{0}, \ldots, T_{n}$ act as in Section 8 , and we let $K_{0}$ act as in Section 6 , We have only to prove the Hecke relations asserted in the theorem. By remark 6.4 we may consider the faithful representation $M=\mathbf{A}$. As in the proof of Proposition 6.8, $K_{0}$ takes the explicit form of Figure 5.

Proposition 9.2. We have the relation $K_{0} \sim \mathrm{q}^{-\rho}$.

Proof. Let $V \in \mathbf{U}$-mod. In Figure 8 , it is proven that the assignment

$$
\begin{gathered}
K: \operatorname{End}_{\mathbb{C}}(V) \rightarrow \operatorname{End}_{\mathbb{C}}(A \otimes V) \\
X \mapsto(X \otimes \mathrm{id} \otimes \mathrm{id}) \circ\left(\operatorname{coev}_{V} \otimes \mathrm{id}\right) \otimes\left(\mathrm{id} \otimes \mathrm{id} \otimes \operatorname{coev} *^{*}\right)
\end{gathered}
$$

is an algebra homomorphism. It follows that $K_{0}=K\left(\left(J^{\rho}\right)^{-1}\right)$ satisfies the same quadratic relation, $K_{0} \sim \mathrm{q}^{-\rho}$, as $\left(J^{\rho}\right)^{-1}$.

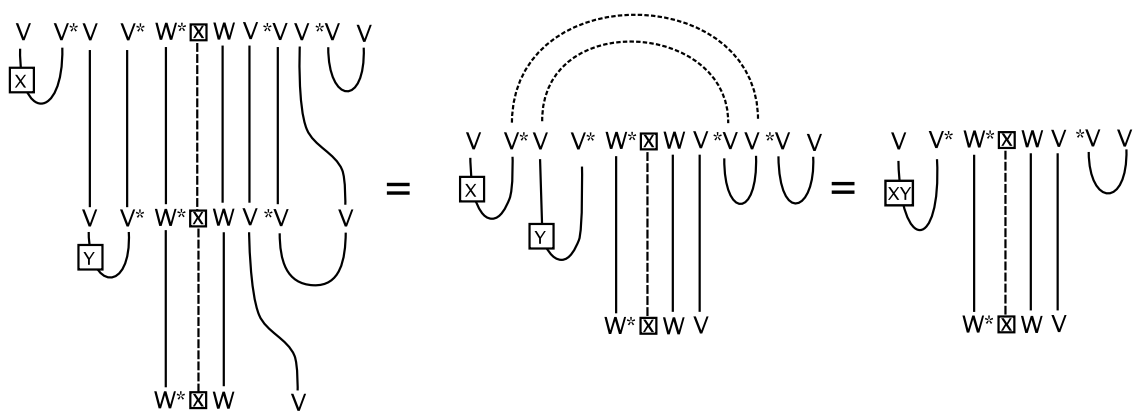

Figure 8. Proof of $K(X) K(Y)=K(X Y)$. The left hand side is the composition $K(X) K(Y)$. The first equality is straightforward. The second equality applies relations (4) to $\operatorname{coev} * V$ as indicated by the dotted lines.

Proposition 9.3. We have the relation $\left(v K_{0} P_{1} T_{0}\right)^{-1} \sim \mathrm{q}^{\nu}$, where $v=\alpha \mathrm{q}^{-\omega}$.

Proof. By definition, we have $v K_{0} P_{1} T_{0}=\mathrm{q}^{-\omega} K_{0} \sigma_{M, V}^{-1} \sigma_{V, M}^{-1}$. We have the following

Lemma 9.4. We have the identity:

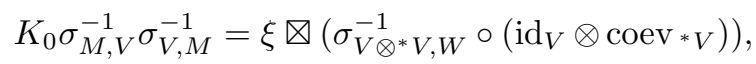

where $\xi=\left(\sigma_{V, W^{*}} \otimes \mathrm{id}\right) \circ\left(\left(J^{\rho}\right)^{-1} \otimes \mathrm{id} \otimes \mathrm{id}\right) \circ\left(\sigma_{W^{*}, V} \otimes \mathrm{id}\right) \circ\left(\mathrm{id} \otimes \operatorname{coev}_{V}\right)$.

Proof. The proof is given in Figure 9

Now, we can express $\xi$ in terms of the $c_{i l}^{\prime}$ :

$$
\begin{aligned}
\xi: f & \mapsto \sum S\left(l_{i j}^{-}\right)\left(J^{\rho}\right)_{j k}^{-1} l_{k l}^{+} f \otimes E_{i}^{l} e_{m} \otimes e^{m} \\
& =\sum c_{i l}^{\prime} f \otimes E_{i}^{l} e_{m} \otimes e^{m},
\end{aligned}
$$

where $\left\{e^{i}\right\}$ denotes the dual basis to $\left\{e_{i}\right\}$. Thus, on the space of $\left(B_{\rho}^{\prime}, \lambda_{\nu}^{\omega}\right)$ invariants, we have

$\xi: \sum f_{j} \otimes w_{j} \otimes v_{j, 1} \otimes \cdots \otimes v_{j, n} \mapsto q^{\omega} \sum f_{j} \otimes\left(J^{\nu}\right)^{-1} e_{m} \otimes e^{m} \otimes w_{j} \otimes v_{j, 1} \otimes \cdots \otimes v_{j, n}$.

Thus, we have that

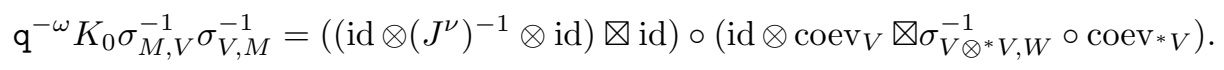




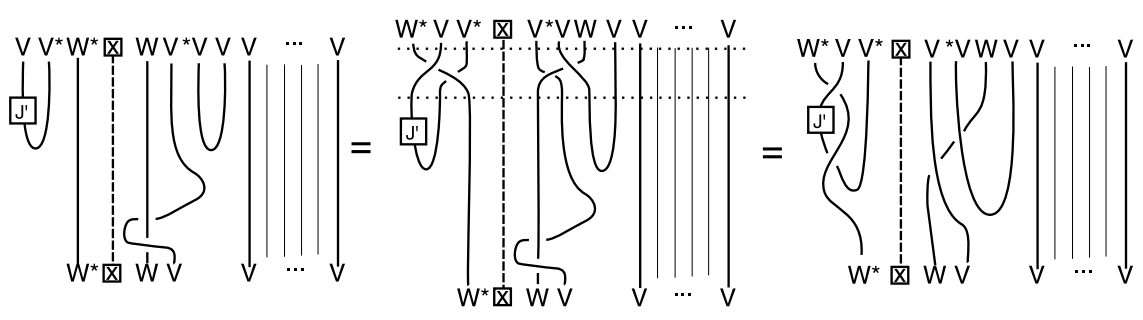

Figure 9. Proof of Lemma 9.4. The first equality applies relations of equation (4) between the dotted lines. The second equality uses only QYBE. We have abbreviated $J^{\prime}:=\left(J^{\rho}\right)^{-1}$.

Now arguing as in Proposition 9.2 we see that $v K_{0} P_{1} T_{0}$ has the same minimal polynomial as $\left(J^{\nu}\right)^{-1}$, and we are done.

The second part of the theorem follows as in the proof of Theorem 8.1

Remark 9.5. A priori, for each $n, N, p, F_{n}^{\sigma, \eta, \tau}$ depends upon the four continuous parameters $\mathrm{q}, \mathrm{q}^{\sigma}, \mathrm{q}^{\eta}, \mathrm{q}^{\tau}$. However, it is clear from the definition that $F_{n}^{\sigma, \eta, \tau}$ is the precomposition of $F_{n}^{\sigma, 0, \tau}$ by the automorphism of $\mathcal{C}$ given by $M \mapsto{ }^{*} \mathbb{1}^{\eta} \otimes M$, corresponding to the fractional tensor power of the determinant character.

A priori, for each $n, N, p, F_{n, \rho, \omega, \nu}^{\sigma, \eta, \tau}$ depends upon the seven continuous parameters, $\mathbf{q}, \mathbf{q}^{\sigma}, \mathbf{q}^{\eta}, \mathbf{q}^{\tau}, \mathbf{q}^{\rho}, \mathbf{q}^{\omega}, \mathbf{q}^{\nu}$. However, as above, we can express $F_{\rho, \omega+\xi, \nu}^{n, \sigma, \eta+\xi, \tau}$ as the precomposition of $F_{n, \rho, \omega, \nu}^{\sigma, \eta, \tau}$ by twisting the $D_{\mathbf{U}}$ module $M$ with a fractional tensor power of the determinant local system. On the other hand, $F_{n, \rho, \omega, \nu}^{\sigma, \eta, \tau}(M)$ will be zero unless $\lambda_{\nu}^{\omega}\left(\operatorname{det}_{\mathrm{q}}\right)=\chi_{\tau}^{\eta}\left(\operatorname{det}_{\mathrm{q}}\right) \mathrm{q}^{-n / N}$. This is because the element $\operatorname{det}_{\mathrm{q}}$ is central and thus its image in $D_{\mathbf{U}}$ under both the left and right actions coincide, so that the values of the characters can only differ by the contribution of the factor $(1 \otimes V)^{\otimes n}$. Thus we really have five continuous parameters.

\section{The Relation to the trigonometric dAHA AND dDAHA}

In this section we recall the construction in [EFM], and show that it may be recovered as the trigonometric degeneration of our construction. Furthermore, we reprove the main results from that paper, quoted below as Theorems 10.1 and 10.2 . Beyond giving a new proof of a known result, this serves two purposes: it provides us an explicit check of our computations in the preceding section, and it also illustrates the process of trigonometric degeneration, whereby very complicated Lie-theoretic formulas appear as the first derivative in $\hbar$ of considerably more natural formulas in quantum groups and braided tensor categories.

10.1. The dAHA of type $B C_{n}$. Let $\mathcal{W}_{n}=\mathcal{S}_{n} \ltimes\left(\mathbb{Z}_{2}\right)^{n}$ be the Weyl group of type $B C_{n}$. We denote by $s_{i j}$ the reflection in this group corresponding to the root $\varepsilon_{i}-\varepsilon_{j}$, and by $\gamma_{i}$ the reflection corresponding to $\varepsilon_{i}$ We abbreviate $s_{i}:=s_{i, i+1}$. The type $B C_{n}$ dAHA $\mathcal{H}_{n}^{\mathrm{deg}}\left(\kappa_{1}, \kappa_{2}\right)$ is generated by $y_{1}, \ldots, y_{n}$ and $\mathbb{C}\left[\mathcal{W}_{n}\right]$, with cross relations:

$$
\begin{array}{r}
s_{i} y_{i}-y_{i+1} s_{i}=\kappa_{1} ; \quad\left[s_{i}, y_{j}\right]=0, \quad \forall j \neq i, i+1 ; \\
\gamma_{n} y_{n}+y_{n} \gamma_{n}=\kappa_{2} ; \quad\left[\gamma_{n}, y_{j}\right]=0, \quad \forall j \neq n ; \quad\left[y_{i}, y_{j}\right]=0 .
\end{array}
$$

For any $c \neq 0$, we have an isomorphism $\mathcal{H}_{n}^{\operatorname{deg}}\left(\kappa_{1}, \kappa_{2}\right) \cong \mathcal{H}_{n}^{\operatorname{deg}}\left(c \kappa_{1}, c \kappa_{2}\right)$. 
Let us recall the construction of the functor $F_{n, p, \mu}$ in [EFM]. Let $\mathbb{C}^{N}$ be the vector representation of $\mathfrak{g}=\mathfrak{g l}_{N}$. Let $M$ be a $\mathfrak{g}$-module. Define

$$
F_{n, p, \mu}(M)=\left(M \otimes\left(\mathbb{C}^{N}\right)^{\otimes n}\right)^{\mathfrak{k}_{0}, \mu},
$$

where $\mathfrak{k}_{0}$ is the subalgebra in $\mathfrak{k}=\mathfrak{g l}_{p} \times \mathfrak{g l}_{q}$ consisting of trace zero elements and, for $\mu \in \mathbb{C},\left(\mathfrak{k}_{0}, \mu\right)$-invariants means for all $x \in \mathfrak{k}_{0}, x v=\mu \chi(x) v$. Here $\chi$ is a character of $\mathfrak{k}$ defined in $[\mathrm{EFM}$ :

$$
\chi\left(\left(\begin{array}{cc}
A_{1} & 0 \\
0 & A_{2}
\end{array}\right)\right)=q \operatorname{tr} A_{1}-p \operatorname{tr} A_{2} .
$$

The Weyl group $\mathcal{W}_{n}$ acts on $F_{n, p, \mu}(M)$ in the following way: the element $s_{i j}$ acts by exchanging the $i$-th and $j$-th factors, and $\gamma_{i}$ acts by multiplying the $i$-th factor by $J=\left(\begin{array}{cc}I_{p} & \\ & -I_{q}\end{array}\right)$.

Define elements $y_{k} \in \operatorname{End}_{\mathbb{C}}\left(F_{n, p, \mu}(M)\right)$ as follows:

$$
y_{i}=-\sum_{s \mid t}\left(E_{s}^{t} \otimes E_{t}^{s}\right)_{0 i}+\frac{p-q-\mu N}{2} \gamma_{i}+\frac{1}{2} \sum_{k>i} s_{i k}-\frac{1}{2} \sum_{k<i} s_{i k}+\frac{1}{2} \sum_{i \neq k} s_{i k} \gamma_{i} \gamma_{k}
$$

where $\sum_{s \mid t}=\sum_{s=1}^{p} \sum_{t=p+1}^{n}+\sum_{t=1}^{p} \sum_{s=p+1}^{n}$, the first component acts on $M$ and the second component acts on the $k$-th factor of the tensor product.

Theorem 10.1 ([EFM]). The above action of $\mathcal{W}_{n}$ and the elements $y_{i}$ define a representation of the degenerate affine Hecke algebra $\mathcal{H}_{n}^{\mathrm{deg}}\left(\kappa_{1}, \kappa_{2}\right)$ on the space $F_{n, p, \mu}(M)$, with

$$
\kappa_{1}=1, \quad \kappa_{2}=p-q-\mu N .
$$

10.2. The dDAHA of type $B C_{n}$. The type $B C_{n}$ dDAHA $\mathcal{H}^{\mathrm{deg}}\left(t, k_{1}, k_{2}, k_{3}\right)$ is generated by two commutative families $\left\{x_{i}, i=1, \ldots, n\right\},\left\{y_{i}, i=1, \ldots, n\right\}$ and $\mathbb{C}\left[\mathcal{W}_{n}\right]$, with relations:

i) $s_{i} x_{i}-x_{i+1} s_{i}=0,\left[s_{i}, x_{j}\right]=0,(j \neq i, i+1)$;

ii) $s_{i} y_{i}-y_{i+1} s_{i}=k_{1},\left[s_{i}, y_{j}\right]=0,(j \neq i, i+1)$;

iii) $\gamma_{n} y_{n}+y_{n} \gamma_{n}=k_{2}+k_{3}, \gamma_{n} x_{n}=x_{n}^{-1} \gamma_{n}$, $\left[\gamma_{n}, y_{j}\right]=\left[\gamma_{n}, x_{j}\right]=0,(j \neq n)$

vi) $\left[y_{j}, x_{i}\right]=k_{1} x_{i} s_{i j}-k_{1} x_{i} s_{i j} \gamma_{i} \gamma_{j}$

v) $\left[y_{i}, x_{j}\right]=k_{1} x_{i} s_{i j}-k_{1} x_{j} s_{i j} \gamma_{i} \gamma_{j},(i<j)$

$$
\begin{gathered}
{\left[y_{i}, x_{i}\right]=t x_{i}-k_{1} x_{i} \sum_{k>i} s_{i k}-k_{1} \sum_{k<i} s_{i k} x_{i}-k_{1} x_{i} \sum_{k \neq i} s_{i k} \gamma_{i} \gamma_{k}} \\
-\left(k_{2}+k_{3}\right) x_{i} \gamma_{i}-k_{2} \gamma_{i} .
\end{gathered}
$$

In particular, we see that the subalgebra in the dDAHA generated by $\mathcal{W}_{n}$ and the $y_{i}$ is $\mathcal{H}_{n}^{\mathrm{deg}}\left(\kappa_{1}, \kappa_{2}\right)$, where $\kappa_{1}=k_{1}$ and $\kappa_{2}=k_{2}+k_{3}$.

Let $\lambda \in \mathbb{C}$. For $x \in \mathfrak{g}$, let $L_{x}$ denote the vector field on $G$ generated by the left action of $x$. Let $D^{\lambda}(\mathrm{GL}(N) /(\mathrm{GL}(p) \times \mathrm{GL}(q)))$ be the sheaf of differential operators on $\mathrm{GL}(N) /(\mathrm{GL}(p) \times \mathrm{GL}(q))$, twisted by the character $\lambda \chi$.

Let $M$ be a $D^{\lambda}(\mathrm{GL}(N) /(\mathrm{GL}(p) \times \mathrm{GL}(q)))$-module. Then $M$ is naturally a $\mathfrak{g}$ module, via the vector fields $L_{x}$. Define

$$
F_{n, p, \mu}^{\lambda}(M)=\left(M \otimes V^{\otimes n}\right)^{\mathfrak{k}_{0}, \mu} .
$$

Then $F_{n, p, \mu}^{\lambda}(M)$ is a $\mathcal{H}_{n}^{\mathrm{deg}}$-module as in the Theorem 10.1 . 
For $i=1, \ldots, n$, define the following linear operators on the space $F_{n, p, \mu}^{\lambda}(M)$ :

$$
x_{i}=\sum_{s, t}\left(A J A^{-1} J\right)_{s t} \otimes\left(E_{s}^{t}\right)_{i}
$$

where $\left(A J A^{-1} J\right)_{s t}$ is the function of $A \in \mathrm{GL}(N) / \mathrm{GL}(p) \times \mathrm{GL}(q)$ which takes the $s t$ -th element of $A J A^{-1} J$ and the second component acts on the $i$-th factor in $V^{\otimes n}$.

Theorem 10.2 ([EFM]). The above action of $\mathcal{W}_{n}$ and the elements $x_{i}, y_{i}$ define a representation of the $d D A H A \mathcal{H L}^{\mathrm{deg}}\left(t, k_{1}, k_{2}, k_{3}\right)$ on the space $F_{n, p, \mu}^{\lambda}(M)$, with

$$
t=\frac{2 n}{N}+(\lambda+\mu)(q-p), \quad k_{1}=1, \quad k_{2}=p-q-\lambda N, \quad k_{3}=(\lambda-\mu) N .
$$

We have a functor $F_{n, p, \mu}^{\lambda}$ from the the category of $D^{\lambda}(\mathrm{GL}(N) / \mathrm{GL}(p) \times \mathrm{GL}(q))$ modules to the category of representations of the type $B C_{n}$ dDAHA with such parameters.

10.3. The trigonometric degeneration of the DAHA. In Ch, Cherednik defined the DDAHA of a root system as a suitable quasi-classical limit of the DAHA. In this section, we explain how to apply this procedure to the DAHA of type $C^{\vee} C_{n}$ to recover the presentation of the dDAHA in Section 10.2 . Thus we take $\mathcal{K}=\mathbb{C}((\hbar))$ in the definitions of Section 2.2

Recall that in $\left[\mathrm{S}\right.$, we have a faithful representation of the DAHA of type $C^{\vee} C_{n}$ which is given by follows. Let $\mathbb{C}[x]=\mathbb{C}\left[x_{1}^{ \pm}, \ldots, x_{n}^{ \pm}\right]$, with the $B C_{n}$ Weyl group acting by by permuting and inverting the $x_{i}$. Define

$$
\begin{aligned}
& \pi\left(X_{i}\right):=x_{i}, \\
& \pi\left(T_{0}\right):=t_{0}+t_{0}^{-1} \frac{\left(1-v t_{0} u_{0} x_{1}^{-1}\right)\left(1+v t_{0} u_{0}^{-1} x_{1}^{-1}\right)}{1-v^{2} x_{1}^{-2}}\left(s_{0}-1\right), \\
& \pi\left(T_{i}\right):=t+t^{-1} \frac{1-t^{2} x_{i} x_{i+1}^{-1}}{1-x_{i} x_{i+1}^{-1}}\left(s_{i}-1\right), \\
& \pi\left(T_{n}\right):=t_{n}+t_{n}^{-1} \frac{\left(1-t_{n} u_{n} x_{n}\right)\left(1+t_{n} u_{n}^{-1} x_{n}\right)}{1-x_{n}^{2}}\left(\gamma_{n}-1\right),
\end{aligned}
$$

for, $i=1, \ldots, n-1$. Then we have

Theorem $10.3([\mathrm{~S}]$, Theorem 3.1, 3.2). The map $\pi$ extends to a faithful representation of the $C^{\vee} C_{n} D A H A$ on $\mathbb{C}[x]$.

Let $m_{1}, \ldots m_{6} \in \mathbb{C}$, and define the following elements of $\mathbb{C}[[\hbar]]$ :

$$
\mathrm{q}=e^{\hbar}, t=\mathrm{q}^{m_{1}}, t_{n}=\mathrm{q}^{m_{2}}, t_{0}=\mathrm{q}^{m_{3}}, u_{0}=\mathrm{q}^{m_{4}}, u_{n}=\mathrm{q}^{m_{5}}, v=\mathrm{q}^{m_{6}}
$$

Let $\mathcal{H}_{\hbar}$ denote the closed subalgebra of $\operatorname{End}_{\mathbb{C}[[\hbar]]}\left(\mathbb{C}\left[x_{1}^{ \pm 1}, \ldots, x_{n}^{ \pm 1}\right][[\hbar]]\right)$ generated by the operators in Theorem 10.3. As the formulas expressing $X_{i}, T_{0}, T_{i}$ and $T_{n}$ in terms of the $x_{i}, s_{0}, s_{i}$, and $s_{n}$ are invertible in $\mathbb{C}[[\hbar]], \mathcal{H}_{\hbar}$ is also generated by the latter set of elements.

Proposition 10.4. The natural map on the (lower-case) generators induces an isomorphism $\mathcal{H}_{\hbar} / \hbar \mathcal{H}_{\hbar} \cong \mathcal{H}^{\mathrm{deg}}\left(t, k_{1}, k_{2}, k_{3}\right)$. 
Proof. By a direct computation, which we omit, it can be seen that the relations of the $C^{\vee} C_{n}$ type DAHA degenerate to the relations in the type $B C_{n}$ degenerate double affine Hecke algebra. The parameter correspondence is given by

$$
k_{1}=m_{1}, k_{2}=m_{2}, k_{3}=m_{3}=m_{4}+m_{5}, t=m_{2}+m_{3}+m_{6} .
$$

10.4. The trigonometric degeneration of $\mathbf{B}_{\sigma}$. In this subsection, we let $\sigma \in \mathbb{C}$, and define the power series

$$
\mathrm{q}:=e^{\hbar}, \quad \mathrm{q}^{\sigma}:=e^{\hbar \sigma} \in \mathbb{C}[[\hbar]] .
$$

In this way the algebras $\mathcal{U}_{\mathrm{q}}(\mathfrak{g})$ and $\mathbf{B}_{\sigma}$ considered throughout become $\mathbb{C}[[\hbar]]$-algebras.

Recall that a $\mathbb{C}[[\hbar]]$-subalgebra $\mathbf{B}$ of a $\mathbb{C}[[\hbar]]$-algebra $\mathbf{A}$ is called saturated if $\hbar a \in \mathbf{B} \Rightarrow a \in \mathbf{B}$. The saturation $\mathbf{B}^{s}$ of $\mathbf{B}$ is the smallest saturated subalgebra containing $\mathbf{B}$. The quasi-classical limit of a saturated subalgebra $\mathbf{B} \subset \mathbf{A}$ is the subalgebra $\mathbf{B} / \hbar \mathbf{B}$ of $\mathbf{A} / \hbar \mathbf{A}$. The following is an elaboration of [DS], Remark 6.4:

Claim 10.5. For all $\sigma \in \mathbb{C}$, the quasi-classical limit of the subalgebra $\mathbf{B}_{\sigma}^{s}$ is $\mathcal{U}\left(\mathfrak{k}^{\prime}\right)$, where $\mathfrak{k}^{\prime}$ is the subalgebra of $\mathfrak{g l}_{N}$ defined in Section 7.4.

Proof. As remarked in the proof of Proposition 7.6, the relations of the reflection equation algebra imply that $\mathbf{B}_{\sigma}$ is spanned over $\mathbb{C}[[\hbar]]$ by ordered monomials in the $c_{i l}$, and therefore its saturation $\mathbf{B}_{\sigma}^{s}$ is a saturated subalgebra whose quasi-classical limit is generated by the quasi-classical limits of the generators $c_{i l}$. Thus it remains only to compute the quasi-classical limits of the $c_{i l}$ and check that they coincide with the generators of $\mathcal{U}\left(\mathfrak{k}^{\prime}\right)$.

We recall the formula for the generators $c_{i l}$ :

$$
c_{i l}=\sum_{j, k=1}^{m} l_{i j}^{+}\left(J_{V}\right)_{j k} S\left(l_{k l}^{-}\right) .
$$

The classical limits of each $l_{i j}^{ \pm}$are $\delta_{i j}$. We recall the well-known formulas for the quasi-classical limits of the $l_{i j}^{ \pm}$:

$$
\begin{gathered}
\lim _{\mathrm{q} \rightarrow 1} \frac{l_{i j}^{ \pm}}{\mathrm{q}-\mathrm{q}^{-1}}=-\lim _{\mathrm{q} \rightarrow 1} \frac{S\left(l_{i j}^{ \pm}\right)}{\mathrm{q}-\mathrm{q}^{-1}}= \pm E_{j}^{i}, \text { for } i \neq j ; \\
\lim _{\mathrm{q} \rightarrow 1} \frac{2\left(l_{i i}^{+}-l_{j j}^{-}\right)}{\mathrm{q}-\mathrm{q}^{-1}}=E_{i}^{i}+E_{j}^{j} .
\end{gathered}
$$

The only terms in the summation expression for $c_{i l}$ which will contribute to the quasi-classical limit are those in which either $i=j$ or $k=l$; in all other cases, the term will vanish to second order in $\hbar$, and thus its quasiclassical limit will be zero. We have six cases to compute, according to the block form of $J^{\sigma}$.

Case 1a: $1 \leq i<l \leq p$.

$$
\begin{aligned}
\lim _{\mathrm{q} \rightarrow 1} \frac{c_{i l}}{\mathrm{q}-\mathrm{q}^{-1}} & =\lim _{\mathrm{q} \rightarrow 1} \frac{l_{i, N-l+1}^{+} S\left(l_{l l}^{-}\right)+l_{i i}^{+} S\left(l_{N-i+1, l}^{-}\right)}{\mathrm{q}-\mathrm{q}^{-1}} \\
& =E_{N-l+1}^{i}+E_{l}^{N-i+1} ;
\end{aligned}
$$


Case 1b: $1 \leq l<i \leq p$.

$$
\begin{aligned}
\lim _{\mathrm{q} \rightarrow 1} \frac{c_{i l}}{\mathrm{q}-\mathrm{q}^{-1}} & =\lim _{\mathrm{q} \rightarrow 1} \frac{l_{i, i}^{+} S\left(l_{N-i+1, l}^{-}\right)+l_{i, N-l+1}^{+} S\left(l_{l, l}^{-}\right)}{\mathrm{q}-\mathrm{q}^{-1}} \\
& =E_{l}^{N-i+1}+E_{N-l+1}^{i} ;
\end{aligned}
$$

Case 1c: $1 \leq i=l \leq p$.

$$
\begin{aligned}
\lim _{\mathrm{q} \rightarrow 1} \frac{c_{i i}}{\mathrm{q}-\mathrm{q}^{-1}} & =\lim _{\mathrm{q} \rightarrow 1} \frac{l_{i i}^{+} S\left(l_{i i}^{-}\right)\left(\mathrm{q}^{\sigma}-\mathrm{q}^{-\sigma}\right)+l_{i, i}^{+} S\left(l_{N-i+1, i}^{-}\right)+l_{i, N-i+1}^{+} S\left(l_{i, i}^{-}\right)}{\mathrm{q}-\mathrm{q}^{-1}} \\
& =\sigma+E_{i}^{N-i+1}+E_{N-i+1}^{i} ;
\end{aligned}
$$

Case 2: $1 \leq i \leq p, p+1 \leq l \leq N-p$.

$$
\begin{aligned}
\lim _{\mathrm{q} \rightarrow 1} \frac{c_{i l}}{\mathrm{q}-\mathrm{q}^{-1}} & =\lim _{\mathrm{q} \rightarrow 1} \frac{l_{i, i}^{+} S\left(l_{N-i+1, l}^{-}\right)-\mathrm{q}^{-\sigma} l_{i, l}^{+} S\left(l_{l, l}^{-}\right)}{\mathrm{q}-\mathrm{q}^{-1}} \\
& =E_{l}^{N-i+1}-E_{l}^{i} ;
\end{aligned}
$$

Case 3a: $N-p+1 \leq l \leq N, 1 \leq i<N-l+1$.

$$
\begin{aligned}
\lim _{\mathrm{q} \rightarrow 1} \frac{c_{i l}}{\mathrm{q}-\mathrm{q}^{-1}} & =\lim _{\mathrm{q} \rightarrow 1} \frac{l_{i, i}^{+} S\left(l_{N-i+1, l}^{-}\right)+l_{i, N-l+1}^{+} S\left(l_{l, l}^{-}\right)}{\mathrm{q}-\mathrm{q}^{-1}} \\
& =E_{l}^{N-i+1}+E_{N-l+1}^{i} ;
\end{aligned}
$$

Case 3b: $N-p+1 \leq l \leq N, i=N-l+1$.

$$
\begin{aligned}
\lim _{\mathrm{q} \rightarrow 1} \frac{2-2 c_{i l}}{\mathrm{q}-\mathrm{q}^{-1}} & =\lim _{\mathrm{q} \rightarrow 1} \frac{2\left(1-l_{i, i}^{+} S\left(l_{N-i+1, N-i+1}^{-}\right)\right)}{\mathrm{q}-\mathrm{q}^{-1}} \\
& =\lim _{\mathrm{q} \rightarrow 1} \frac{2\left(l_{N-i+1, N-i+1}^{-}-l_{i, i}^{+}\right) S\left(l_{N-i+1, N-i+1}^{-}\right)}{\mathrm{q}-\mathrm{q}^{-1}} \\
& =-E_{N-i+1}^{N-i+1}-E_{i}^{i} ;
\end{aligned}
$$

Case 4: $1 \leq l \leq p, p+1 \leq i \leq N-p$.

$$
\begin{aligned}
\lim _{\mathrm{q} \rightarrow 1} \frac{c_{i l}}{\mathrm{q}-\mathrm{q}^{-1}} & =\lim _{\mathrm{q} \rightarrow 1} \frac{-\mathrm{q}^{-\sigma} l_{i, i}^{+} S\left(l_{i, l}^{-}\right)+l_{i, N-l+1}^{+} S\left(l_{l, l}^{-}\right)}{\mathrm{q}-\mathrm{q}^{-1}} \\
& =E_{N-l+1}^{i}-E_{l}^{i} ;
\end{aligned}
$$

Case 5a: $p+1 \leq i<l \leq N-p$.

$$
\lim _{\mathrm{q} \rightarrow 1} \frac{c_{i l}}{\mathrm{q}-\mathrm{q}^{-1}}=\lim _{\mathrm{q} \rightarrow 1} \frac{\mathrm{q}^{-\sigma} l_{i, l}^{+} S\left(l_{l, l}^{-}\right)}{\mathrm{q}-\mathrm{q}^{-1}}=E_{l}^{i}
$$

Case 5b: $p+1 \leq i=l \leq N-p$.

$$
\begin{aligned}
\lim _{\mathrm{q} \rightarrow 1} \frac{\mathrm{q}^{-\sigma}+c_{i i}}{\mathrm{q}-\mathrm{q}^{-1}} & =\lim _{\mathrm{q} \rightarrow 1} \frac{\mathrm{q}^{-\sigma}-\mathrm{q}^{-\sigma} l_{i, i}^{+} S\left(l_{i, i}^{-}\right)}{\mathrm{q}-\mathrm{q}^{-1}} \\
& =\lim _{\mathrm{q} \rightarrow 1} \frac{\mathrm{q}^{-\sigma}\left(l_{i, i}^{-}-l_{i, i}^{+}\right) S\left(l_{i, i}^{-}\right)}{\mathrm{q}-\mathrm{q}^{-1}} \\
& =-E_{i}^{i} ;
\end{aligned}
$$


Case 5c: $p+1 \leq l<i \leq N-p$.

$$
\lim _{\mathrm{q} \rightarrow 1} \frac{c_{i l}}{\mathrm{q}-\mathrm{q}^{-1}}=\lim _{\mathrm{q} \rightarrow 1} \frac{-\mathrm{q}^{-\sigma} l_{i, i}^{+} S\left(l_{i, l}^{-}\right)}{\mathrm{q}-\mathrm{q}^{-1}}=-E_{l}^{i} ;
$$

Case 6a: $N-p+1 \leq i \leq N, 1 \leq l<N-i+1$.

$$
\begin{aligned}
\lim _{\mathrm{q} \rightarrow 1} \frac{c_{i l}}{\mathrm{q}-\mathrm{q}^{-1}} & =\lim _{\mathrm{q} \rightarrow 1} \frac{l_{i, i}^{+} S\left(l_{N-i+1, l}^{-}\right)+l_{i, N-l+1}^{+} S\left(l_{l, l}^{-}\right)}{\mathrm{q}-\mathrm{q}^{-1}} \\
& =E_{l}^{N-i+1}+E_{N-l+1}^{i} ;
\end{aligned}
$$

Case 6b: $N-p+1 \leq i \leq N, l=N-i+1$.

$$
\begin{aligned}
\lim _{\mathrm{q} \rightarrow 1} \frac{2-2 c_{i l}}{\mathrm{q}-\mathrm{q}^{-1}} & =\lim _{\mathrm{q} \rightarrow 1} \frac{2\left(1-l_{i, i}^{+} S\left(l_{N-i+1, N-i+1}^{-}\right)\right)}{\mathrm{q}-\mathrm{q}^{-1}} \\
& =\lim _{\mathrm{q} \rightarrow 1} \frac{2\left(l_{N-i+1, N-i+1}^{-}-l_{i, i}^{+}\right) S\left(l_{N-i+1, N-i+1}^{-}\right)}{\mathrm{q}-\mathrm{q}^{-1}} \\
& =-E_{i}^{i}-E_{N-i+1}^{N-i+1} .
\end{aligned}
$$

Finally, we let

$$
g=\sum_{k=1}^{p} E_{k}^{k}-\sum_{k=p+1}^{n} E_{k}^{k}+\sum_{k=1}^{p} E_{n-k+1}^{k}+\sum_{k=1}^{p} E_{k}^{n-k+1}
$$

and conjugate each of the above elements by $g$. We have

$$
\begin{aligned}
g\left(E_{N-l+1}^{i}+E_{l}^{N-i+1}\right) g^{-1} & =E_{l}^{i}-E_{N-l+1}^{N-i+1}, \text { in Case 1a; } \\
g\left(E_{l}^{N-i+1}+E_{N-l+1}^{i}\right) g^{-1} & =E_{l}^{i}-E_{N-l+1}^{N-i+1}, \quad \text { in Case } 1 \mathrm{~b} ; \\
\sigma+g\left(E_{i}^{N-i+1}+E_{N-i+1}^{i}\right) g^{-1} & =\sigma+E_{i}^{i}-E_{N-i+1}^{N-i+1}, \quad \text { in Case } 1 \mathrm{c} ; \\
g\left(E_{l}^{N-i+1}-E_{l}^{i}\right) g^{-1} & =E_{l}^{N-i+1}, \quad \text { in Case } 2 ; \\
g\left(E_{l}^{N-i+1}+E_{N-l+1}^{i}\right) g^{-1} & =E_{l}^{N-i+1}+E_{N-l+1}^{i}, \quad \text { in Case } 3 \mathrm{a} ; \\
g\left(-E_{i}^{i}-E_{N-i+1}^{N-i+1}\right) g^{-1} & =-E_{i}^{i}-E_{N-i+1}^{N-i+1}, \quad \text { for Cases } 3 \mathrm{~b} \text { and } 6 \mathrm{~b} ; \\
g\left(E_{N-l+1}^{i}-E_{l}^{i}\right) g^{-1} & =2 E_{N-l+1}^{i}, \text { in Case } 4 ; \\
g\left(E_{l}^{i}\right) g^{-1} & =E_{l}^{i}, \quad \text { in Cases } 5 \mathrm{a}, \mathrm{b} \text { and c; } \\
g\left(E_{l}^{N-i+1}+E_{N-l+1}^{i}\right) g^{-1} & =E_{l}^{N-i+1}+E_{N-l+1}^{i}, \quad \text { in Case } 6 \mathrm{a} ;
\end{aligned}
$$

Thus we see by direct inspection that the quasi-classical limit of the subalgebra $\mathbf{B}_{\sigma}$ is the algebra $\mathcal{U}\left(\mathfrak{k}^{\prime}\right)$.

10.5. The trigonometric degeneration of the character $\chi_{\tau}^{\eta}$. By trigonometric degeneration of a character $\chi: \mathbf{B}_{\sigma} \rightarrow \mathbb{C}$ we will mean the following: first we work over $\mathbb{C}[[\hbar]]$, and set $\mathbf{q}=e^{\hbar}, \mathbf{q}^{\sigma}=e^{\hbar \sigma}$. We thus view $\chi$ as a homomorphism to $\mathbb{C}[[\hbar]]$ instead. We send $a \in \mathbf{B}_{\sigma}^{s} / \hbar \mathbf{B}_{\sigma}^{s}$ to $\chi(\hat{a}) \bmod \hbar$ for any lift $\hat{a}$ of $a$.

We now apply the explicit computations above to compute the trigonometric degeneration of the characters $\chi_{\tau}^{\eta}$. In order to be compatible with the conventions of [EFM], we will consider the character $\tilde{\chi}_{\tau}^{\eta}: \mathfrak{g l}_{p} \times \mathfrak{g l}_{q} \rightarrow \mathfrak{k}^{\prime} \rightarrow \mathbb{C}$, obtained by precomposing with conjugation by $g^{-1}$, and applying the quasi-classical limit of the character $\chi_{\tau}^{\eta}: \mathbf{B}_{\sigma}^{s} \rightarrow \mathbb{C}$. We compute that: 


$$
\tilde{\chi}_{\tau}^{\eta}\left(\left(\begin{array}{cc}
A_{1} & 0 \\
0 & A_{2}
\end{array}\right)\right)=\frac{\eta+\tau-\sigma}{2} \operatorname{tr} A_{1}+\frac{\eta+\sigma-\tau}{2} \operatorname{tr} A_{2} .
$$

Thus, we have that

$$
\tilde{\chi}_{\tau}^{\eta}=\left(\frac{\eta}{2}+\frac{(p-q)(\tau-\sigma)}{2 N}\right) \operatorname{tr}+\frac{(\tau-\sigma)}{N} \chi
$$

where $\chi$ is that from equation (31).

Similarly, we can compute the character $\tilde{\lambda}_{\nu}^{\omega}: \mathfrak{g l}_{p} \times \mathfrak{g l}_{q} \rightarrow \mathbb{C}$ obtained from $\lambda_{\nu}^{\omega}$ by quasi-classical limit. We have

$$
\tilde{\lambda}_{\nu}^{\omega}=\left(\frac{\omega}{2}+\frac{(p-q)(\rho-\nu)}{2 N}\right) \operatorname{tr}+\frac{(\rho-\nu)}{N} \chi .
$$

10.6. An alternate presentation for the DAHA. In this section, we recall an alternate presentation for the DAHA (e.g. [S], EGO]), and prove that it coincides with Definition 2.3.

Let $[a, b]$ denote the set of integers between $a$ and $b$ inclusive, regardless of which is larger. Recall the elements $T_{(i \cdots j)}$ and $P_{i}$ from Section 2. By direct computation, we have the following:

Lemma 10.6. We have the following relations:

$$
\begin{gathered}
T_{(i \cdots j)} T_{(k \cdots l)}=\left\{\begin{array}{cc}
T_{(k \cdots l)} T_{(i \cdots j)}, & {[i, j] \cap[k, l]=\emptyset,} \\
T_{(k \cdots l)} T_{(i+1 \cdots j+1)}, & {[i, j] \subsetneq[k, l], k>l,} \\
T_{(k \cdots l)} T_{(i-1 \cdots j-1)}, & {[i, j] \subsetneq[k, l], k<l,}
\end{array}\right. \\
T_{i} P_{i+1} T_{i}=P_{i}, \quad T_{i} P_{j}=P_{j} T_{i} \quad(j \neq i, i+1), \\
P_{i} P_{j}=P_{j} P_{i}, \quad i, j=1, \ldots, n-1 .
\end{gathered}
$$

Consider the following elements:

$$
\begin{aligned}
Y_{i} & :=P_{i} T_{(i \cdots 1)} T_{0} T_{(i \cdots 1)}^{-1}, \\
X_{i} & :=P_{i}^{-1} T_{(1 \cdots i)}^{-1} K_{0}^{-1} T_{(1 \cdots i)} .
\end{aligned}
$$

Proposition 10.7. $\widetilde{\mathcal{B}}_{n}$ is generated by the group $\mathcal{B}_{n}$ and elements $X_{1}, \ldots, X_{n}$, $Y_{1}, \ldots Y_{n}$, with the relations:

$$
\begin{gathered}
T_{i} Y_{i+1} T_{i}=Y_{i}, T_{i} X_{i} T_{i}=X_{i+1}, X_{i} X_{j}=X_{j} X_{i}, Y_{i} Y_{j}=Y_{j} Y_{i}(i, j=1, \ldots, n), \\
T_{i} Y_{j}=Y_{j} T_{i}, T_{i} X_{j}=X_{j} T_{i}(j \neq i, i+1), T_{n} Y_{n-1}=Y_{n-1} T_{n}, T_{n} X_{n-1}=X_{n-1} T_{n}, \\
X_{i}\left(P_{1}^{-1} Y_{1}\right)=\left(P_{1}^{-1} Y_{1}\right) X_{i}(i=2, \ldots, n-1) .
\end{gathered}
$$

Proof. Let $\widetilde{\mathcal{B}^{\prime}}$ denote the group specified in the proposition, and reserve $\widetilde{\mathcal{B}}$ for the group given by Definition 2.3. We define $\phi: \widetilde{\mathcal{B}^{\prime}} \rightarrow \widetilde{\mathcal{B}}$ on generators:

$$
\begin{aligned}
\phi: \quad & T_{i} \mapsto T_{i}, \quad i=1, \ldots, n, \\
& X_{i} \mapsto P_{i}^{-1} T_{(1 \cdots i)}^{-1} K_{0}^{-1} T_{(1 \cdots i)}, \quad i=1, \ldots, n, \\
& Y_{i} \mapsto P_{i} T_{(i \cdots 1)} T_{0} T_{(i \cdots 1)}^{-1}, \quad i=1, \ldots, n .
\end{aligned}
$$

We leave it to the reader to verify that $\phi$ defines an isomorphism. 
Remark 10.8. Along the lines of Remark 2.5. the isomorphism $\phi$ admits the following geometric interpretation: every elliptic curve $E=\mathbb{C} / \Lambda$ admits a $\mathbb{Z}_{2}$ action, $z \mapsto-z$. Let $E^{\circ}$ denote the complement of the fixed points. It is easy to see that $E^{\circ} / \mathbb{Z}_{2}$ is homeomorphic to $\mathbb{P}^{1} \backslash\left\{p_{1}, p_{2}, p_{3}, p_{4}\right\}$. The generators $X_{i}$ and $Y_{j}$ of $\widetilde{\mathcal{B}}_{n}$ correspond to the horizontal and vertical cycles on $E$, as in [J], Figure 1. The generators $T_{0}, T_{n}$, and $K_{0}$ correspond to loops around $p_{1}, p_{2}$, and $p_{3}$, respectively, so that $\left(K_{0} P_{1} T_{0}\right)^{-1}$ corresponds to a loop around $p_{4}$.

Corollary 10.9. The double affine Hecke algebra is a quotient of $\mathcal{K}[\widetilde{\mathcal{B}}]$ by the relations:

$$
\begin{gathered}
Y_{n} T_{n}^{-1} \sim t_{0}, \quad T_{n} \sim t_{n}, \quad X_{n}^{-1} T_{n}^{-1} \sim u_{n}, \\
v^{-1} Y_{1}^{-1} P_{1} X_{1} \sim u_{0}, \quad T_{i} \sim t(i=1, \ldots, n-1) .
\end{gathered}
$$

Remark 10.10. The operators $T_{0}$ defined in Section 5.2 determine operators $Y_{i}$, via the isomorphism asserted in Proposition 10.7. It should be noted that these coincide with the inverse of the operators $Y_{i}$ which appeared in [J] for the $A_{n-1}$ construction, except that those involved $\mathfrak{s l}_{N}$, rather than $\mathfrak{g l}_{N}$.

10.7. The quasi-classical limit of Theorems $\mathbf{9 . 1}$ and $\mathbf{8 . 1}$. In this section, we compute the quasi-classical limits of the operators appearing in Theorems 9.1 and 8.1. making use of the alternate presentation for the $C^{\vee} C_{n}$ DAHA from the previous section. By comparing the results with the operators in EFM], we can give a reproof of Theorems 10.1 and 10.2 This serves as a consistency check for both papers.

It is well known that the quasi-classical limit of the $R$-matrix of $\mathcal{U}_{\mathrm{q}}\left(\mathfrak{g l}_{N}\right)$ is

$$
1+\hbar r \bmod \hbar^{2}
$$

where $r$ denotes the classical $R$-matrix for $\mathfrak{g l}_{N}$. Thus, for $i=1 \ldots, n-1$, the quasi-classical limit of $T_{i}$ is

$$
s_{i}\left(1+\hbar r_{i, i+1}\right) \bmod \hbar^{2} .
$$

By direct computation, the classical limit of $T_{n}$ is

$$
J^{\prime}+\hbar \sigma \hat{J} \bmod \hbar^{2}
$$

where $\hat{J}=2 \sum_{i \leq p} E_{i}^{i}+\sum_{p+1 \leq i \leq q} E_{i}^{i}$, and $J^{\prime}$ is the classical matrix from equation (25).

Lemma 10.11. When $\mathbf{U}=\mathcal{U}\left(\mathfrak{g l}_{N}\right)$, the operator $K_{0}$ acts as $\left(A J A^{-1}\right)_{j}^{i} \otimes E_{i}^{j}$.

Proof. The proof is by direct computation in the symmetric category $\mathcal{U}(\mathfrak{g})$-mod, and relies on the triviality of the braiding to simplify $K_{0}$. We may choose a basis diagonalizing $J$, and rewrite equation 19 in coordinates, ignoring appearance of $R$-matrices, identifying ${ }^{*} V \cong V^{*}$ canonically, and noting that the classical limit (in 
this basis) of $J^{\sigma}$ is $J$ :

$$
\begin{aligned}
K_{0} & =\sum c_{J v_{k} \otimes v^{k}, v_{j} \otimes v^{i}} \otimes E_{i}^{j} \\
& =\sum c_{v^{k}, v_{j}} c_{J v_{k} \otimes v^{i}} \otimes E_{i}^{j} \\
& =\sum c_{v^{k}, v_{j}} J_{k}^{l} S\left(c_{v^{i}, v_{l}}\right) \otimes E_{i}^{j} \\
& =\sum a_{j}^{k} J_{k}^{l} S\left(a_{l}^{i}\right) \otimes E_{i}^{j} \\
& =\sum\left(A J A^{-1}\right)_{j i} \otimes E_{i}^{j} .
\end{aligned}
$$

Proposition 10.12. The classical limit of $X_{1}$ is $\sum\left(A J A^{-1} J\right)_{j}^{i} \otimes E_{i}^{j}$

Proof. We have $X_{1}=P_{1}^{-1} K_{0}^{-1}$. The classical limit of $P_{1}^{-1}$ is $J_{1}$, by direct computation, using triviality of the braiding, and the fact that $J=J^{-1}$. Thus, by the lemma, we have:

$$
X_{1}=\sum\left(A J A^{-1}\right)_{j}^{i} \otimes J_{k l} E_{k}^{l} E_{i}^{j}=\sum\left(A J A^{-1} J\right)_{j}^{k} \otimes E_{k}^{j},
$$

as desired.

Define $\hat{y}_{i} \in \operatorname{End}_{\mathbb{C}}\left(M \otimes V^{\otimes n}\right)$ by the equation $Y_{i} \equiv 1+\hbar \hat{y}_{i}\left(\bmod \hbar^{2}\right)$. As noted in Remark 10.10, the operators $Y_{i}$ determined by our choice for $T_{0}$ and Proposition 10.7 coincide with the inverse of those of [J]. In order to prove Theorem 8.1, we rescaled $T_{0}$ and thus $Y_{1}$ by $\mathrm{q}^{\eta-N}$ and thus the quasi-classical limit of $y_{1}$ is computed by:

Proposition 10.13 (see [J], Proposition 6.14). The operator $\hat{y}_{1}$ is given by

$$
\hat{y}_{i}=-\Omega_{0 i}-\sum_{j<i} s_{i j}+\frac{\eta-N}{2},
$$

where is the $\Omega=\sum_{i, j} E_{i}^{j} \otimes E_{j}^{i} \in \operatorname{Sym}^{2}(\mathfrak{g})^{\mathfrak{g}}$ is the Casimir element for $\mathfrak{g}=\mathfrak{g l}_{N}$.

The following proposition allows us to compare $\hat{y}_{i}$ with the operators $y_{i}$ from Section 10.1. We have:

Proposition 10.14. As an operator on the $\left(\mathfrak{k}, \tilde{\chi}_{\tau}^{\eta}\right)$-invariants, we have

$$
y_{1}=-\Omega_{01}+\frac{\eta-N}{2}+\frac{(\tau-\sigma)-\mu N}{2} \gamma_{1}
$$

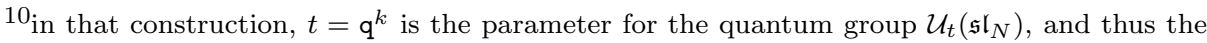
factor $k$ multiplies $\tilde{y}_{i}$. Also, since we work with $\mathfrak{g l}_{N}$, there is not the shift $\frac{i-1}{N}$, which occurs in Proposition 6.14 of [J], because $\Omega^{\mathfrak{s l}}{ }_{N}=\Omega^{\mathfrak{g l}}{ }_{N}-\frac{1}{N} \operatorname{id}_{N} \otimes \operatorname{id}_{N}$
} 
Proof. Recall the summation convention $\sum_{i j}:=\sum_{i, j=1}^{p}+\sum_{i, j=p+1}^{N}$ from [EFM]. First, we set $i=1$ in equation (32), and simplify the summations over $k$ :

$$
\begin{aligned}
S= & \frac{1}{2} \sum_{k>1} s_{1 k}+\frac{1}{2} \sum_{k>1} s_{1 k} \gamma_{1} \gamma_{k} \\
= & \frac{1}{2} \sum_{k>1} \sum_{i, j}\left(E_{i}^{j}\right)_{1} \otimes\left(E_{j}^{i}\right)_{k}+\frac{1}{2} \sum_{k>1} \sum_{i, j}\left(E_{i}^{j} J\right)_{1} \otimes\left(E_{j}^{i} J\right)_{k} \\
= & \sum_{k>1} \sum_{i, j}\left(E_{i}^{j}\right)_{1} \otimes\left(E_{j}^{i}\right)_{k} \\
& \text { (applying the } \left.\tilde{\chi}_{\tau}^{\eta} \text {-invariant property, to the tensor factors in } \mathfrak{k}\right) \\
= & \sum_{i, j}\left(E_{i}^{j}\right)_{1} \tilde{\chi}_{\tau}^{\eta}\left(E_{j}^{i}\right)-\sum_{i, j}\left(E_{i}^{j}\right)_{0} \otimes\left(E_{j}^{i}\right)_{1}-p \sum_{i \leq p}\left(E_{i}^{i}\right)_{1}-q \sum_{i>p}\left(E_{i}^{i}\right)_{1} \\
= & \frac{\eta}{2}+\frac{\tau-\sigma}{2}\left(\sum_{i \leq p}\left(E_{i}^{i}\right)_{1}-\sum_{i>p}\left(E_{i}^{i}\right)_{1}\right)-\sum_{i, j}\left(E_{j}^{i}\right)_{0} \otimes\left(E_{j}^{i}\right)_{1} \\
& \quad-p \sum_{i \leq p}\left(E_{i}^{i}\right)_{1}-q \sum_{i>p}\left(E_{i}^{i}\right)_{1} .
\end{aligned}
$$

Thus, we may rewrite equation 32 :

$$
y_{1}=-\sum_{i, j}\left(E_{i}^{j}\right)_{0} \otimes\left(E_{j}^{i}\right)_{1}+\frac{\eta-N}{2}+\frac{(\tau-\sigma)-\mu N}{2} \gamma_{1} .
$$

Finally, let:

$$
\begin{aligned}
\sigma & =p-q-\lambda N \\
\tau & =(\mu-\lambda) N+p-q \\
\nu-\rho & =(\lambda-\mu) N \\
\eta-\omega & =N+\frac{2 n}{N}+\lambda(q-p)-2 \mu p
\end{aligned}
$$

Comparing with (34), we see that $k_{1}, k_{2}, k_{3}$ and $t$ from the degeneration of the DAHA agree with the parameters of Theorem 10.2. On the other hand, we have shown that the coideal subalgebras $\mathbf{B}_{\sigma}$ and $\mathbf{B}_{\rho}^{\prime}$ both degenerate to the subalgebra $\mathcal{U}\left(\mathfrak{g l}_{p} \times \mathfrak{g l}_{q}\right)$, while the characters $\tilde{\chi}_{\tau}^{\eta}$ and $\tilde{\lambda}_{\nu}^{\rho}$ degenerate to the characters $\mu \chi$ and $(\mu-\lambda) \chi$, respectively, upon restriction to $\mathfrak{g l}_{p} \times \mathfrak{g l}_{q}$.

Thus we may recover Theorems 10.1 and 10.2 as follows. $\eta$ records the spectrum of the center of $\mathfrak{g l}_{N}$ on $M$, which is discarded in [EFM], who consider instead $\mathfrak{s l}_{N}$. Thus by summing the $F_{n}^{\sigma, \eta, \tau}(M)$ over all $\eta$, and $F_{n, \rho, \omega, \nu}^{\sigma, \eta, \tau}(M)$ over all $\eta$ and $\omega$, we recover the spaces of Theorems 10.1 and $10.2{ }^{11}$, respectively as quasi-classical limits. We have shown that the operators $X_{i}$ and $T_{j}$ degenerate to $x_{i}$ and $s_{j}$, respectively, for $i, j=1, \ldots n$, and we have shown that $\hat{y}_{i}=y_{i}$. Thus the entire constructions of [EFM] are recovered as quasi-classical limits of the present results.

\footnotetext{
${ }^{11}$ In that paper, the authors consider $\lambda \chi$-twisted $D$-modules, and $\mu$-invariants. This coincides with $\lambda \chi$-ad-invariants, and $\mu \chi$ left-invariants, or equivalently $(\mu-\lambda) \chi$ right-invariants and $\mu \chi$ left-invariants.
} 


\section{REFERENCES}

[AS] T. Arakawa, T. Suzuki, Duality between $\mathfrak{s l}_{n}(\mathbb{C})$ and the degenerate affine Hecke algebra, Journal of Algebra 209, Academic Press, 1998.

[BK] B. Bakalov, A. Kirillov, Lectures on tensor categories and modular functors, University Lecture Series, 21. American Mathematical Society, Providence, RI, 2001.

[Br] E. Brieskorn, Die Fundamentalgruppe des Raumes der regulren Orbits einer endlichen komplexen Spiegelungsgruppe, Inventiones Mathematicae 12 (1971), no 1, 57-61.

[CEE] D. Calaque, B. Enriquez, P. Etingof, Universal KZB equations I: the elliptic case, Preprint arXiv:math/0702670

[Cal] P. Caldero, Éléments ad-finis de certains groupes quantiques, C. R. Acad. Sci. Paris Sér. I Math. 316 (1993), no. 4, 327-329.

[Ch] I. Cherednik, Double Affine Hecke Algebras, London Math. Soc. Lecture Notes Series 319.

[De1] P. Deligne, Catégories Tannakiennes, In the Grothendieck Festschrift, Vol. II, Prog. Math. 87 (1990), 111-195.

[De2] P. Deligne Catégories tensorielles, (French) Dedicated to Yuri I. Manin on the occasion of his 65th birthday, Mosc. Math. J. 2 (2002), no 2, 227-248.

[DKM] J. Donin, P.P. Kulish, A.I. Mudrov, On a universal solution to the reflection equation, Lett. Math. Phys. 63 (2003), 179-194.

[DM1] J. Donin, A.I. Mudrov, Method of quantum characters in equivariant quantization, Commun. Math. Phys. 234 (2003), 533-555.

[DM2] J. Donin, A.I. Mudrov, Reflection equation, twist, and equivariant quantization, Isreal J. Math. 136 (2003) 11-28.

[Dri] V. Drinfeld, Degenerate affine Hecke algebras and Yangians (Russian), Funktsional. Anal. i Prilozhen. 20 (1986), no. 1.

[DNS] M. Dijkhuizen, M. Noumi, T. Sugitani, Multivariable Askey-Wilson polynomials and quantum complex Grassmannians, Special Functions, $q$-series and related topics 167-177, Fields Inst. Communi., 14.

[DS] M. Dijkhuizen, J. Stokman, Some limit transitions between BC type orthogonal polynomials interpreted on quantum complex Grassmannians, Publ. Res. Inst. Math. Sci. 35 (1999), no. 3, 451-500.

[EFM] P. Etingof, R. Freund, X. Ma, A Lie-theoretic construction of some representations of the degenerate affine and double affine Hecke algebras of type $B C_{n}$, Represent. Theory 13 (2009), 33-49.

[EGO] P. Etingof, W.L. Gan, A. Oblomkov, Generalized double affine Hecke algebras of higher rank, Journal für die reine und angewandte Mathematik (Crelles Journal). Volume 2006, Issue 600, 177-201, 2006.

[EO] P. Etingof, V. Ostrik, Finite tensor categories, Mosc. Math. J., 4:3 (2004), 782-783.

[IS] B. Ion, S. Sahi, Triple groups and Cherednik algebras, Contemp. Math. 417 (2006), 183-206, math.QA/0304186

[J] D. Jordan, Quantum D-modules, elliptic braid groups, and double affine Hecke algebras, IMRN 2009; Vol. 2009: rnp012, 24 pages, doi:10.1093/imrp/rnp012.

[JL] A. Joseph, G. Letzter, Separation of variables for quantized enveloping algebras, Amer. J. Math. 116 (1994), no. 1, 127-177.

[K] C. Kassel, Quantum groups, Graduate Texts in Mathematics, 155. Springer-Verlag, New York, 1995.

[KlSch] A. Klimyk, K. Schmudgen, Quantum groups and their representations, Springer, 1997.

[KoSt] S. Kolb, J. Stokman, Reflection equation algebras, coideal subalgebras, and their centres, Selecta Math. (N.S.) 15 (2009), no. 4, 621-664.

[Kol] S. Kolb, Quantum symmetric pairs and the reflection equation, Algebr. Represent. Theory 11 (2008), no. 6, 519-544.

[L1] G. Letzter, Coideal subalgebras and quantum symmetric pairs, New directions in Hopf algebras, 117-165, Math. Sci. Res. Inst. Publ., 43, Cambridge Univ. Press, Cambridge, 2002.

[L2] G. Letzter, Quantum symmetric pairs and their zonal spherical functions, Transform. Groups 8 (2003), no. 3, 261-292.

[L3] G. Letzter, Harish Chandra modules for quantum symmetric pairs, Representation Theory 4 (2000), 64-96.

[Lus] G. Lusztig, Affine Hecke algebras and their graded version, J. A.M.S. 2 (1989), 599-635. 
[Maj] S. Majid, Foundations of Quantum Group Theory, Cambridge University Press, 2000

[Mud] A, Mudrov, Characters of $\mathcal{U}_{\mathrm{q}}(\mathfrak{g l}(n))$-reflection equation algebra, Lett. Math. Phys. 60 (2002), 283-291.

[N] M. Noumi, Macdonald's symmetric polynomials as zonal spherical functions on some quantum homogeneous spaces, Adv. Math. 123 (1996) 16-77.

[NS] M. Noumi,T. Sugitani, Quantum symmetric spaces and related q-orthogonal polynomials, Group theoretical methods in physics (Singapore) (A. Arima et. al. ed.) World Scientific, 1995, pp. 28-40.

[OS] A. Oblomkov, J. Stokman, Vector valued spherical functions and Macdonald-Koornwinder polynomials, Compos. Math. 141 (2005), no. 5, 1310-1350.

[S] S. Sahi, Nonsymmetric Koornwinder polynomials and duality, Ann. of Math. (2) 150 (1999), no. $1,267-282$.

[tD] T. tom Dieck, Categories of rooted cylinder ribbons and their representations, J. reine angew. Math. 494 (1998), 36-63.

[tDHO] T. tom Dieck, R. Häring-Oldenburg, Quantum groups and cylinder braiding, Forum Math 10 (1998), no. 5, 619-639.

[VV] M. Varagnolo, E. Vasserot, Double affine Hecke algebras at roots of unity, Preprint, arXiv:math/0603744

Department of Mathematics, Massachusetts Institute of Technology, Cambridge, MA 02139, USA

E-mail address: djordan@math.mit.edu

Department of Mathematics, Massachusetts institute of Technology, Cambridge, MA 02139, USA

E-mail address: xma@math.mit.edu 